\title{
The mechanisms underlying synaptic transmission at the layer 4 of sensory cortical areas
}

\author{
$\mathrm{PhD}$ Thesis \\ in partial fulfillment of the requirements \\ for the degree 'Doctor of Philosophy (Ph.D.)' \\ in the Neuroscience Program \\ at the Georg-August-University Göttingen, \\ Faculty of Biology \\ submitted by \\ Chao-Hua Huang \\ born in \\ Kaohsiung, Taiwan
}




\section{Declaration}

I hereby declare that my $\mathrm{PhD}$ thesis 'The mechanisms underlying synaptic transmission

at the layer 4 of sensory cortical areas' has been written independently with no other aids or sources than quoted.

Göttingen, September 20th 2010 



\section{Contents}

\section{Introduction .................................................................................................}

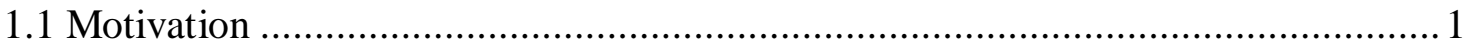

1.2 Top-down, system approaches to the visual function ......................................... 1

1.3 Bottom-up, cellular approaches to the visual function............................................. 3

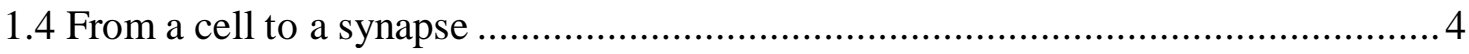

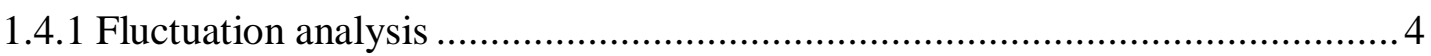

1.4.2 "one-site, one-vesicle" hypothesis v.s. multivesicular release .......................... 8

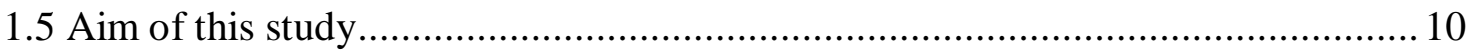

2. Material and Methods .....................................................................11

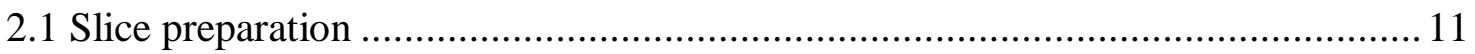

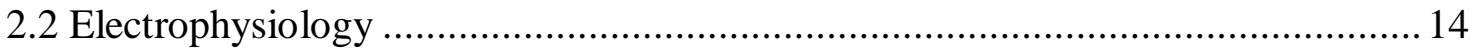

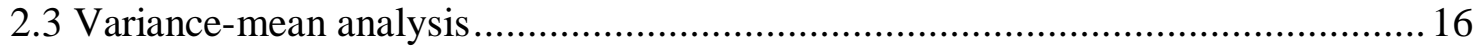

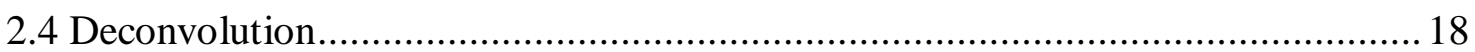

2.5 Dynamic clamp experiments ........................................................................ 19

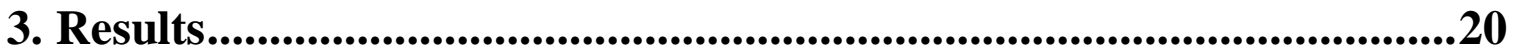

3.1 Variance-mean analysis and the mEPSC amplitude measurement at L4 neurons in

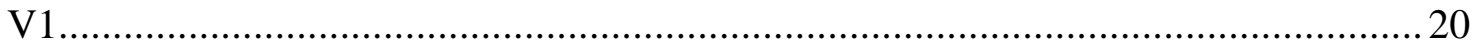

3.2 Estimation of N, Pr, and q under physiological condition with variance-mean

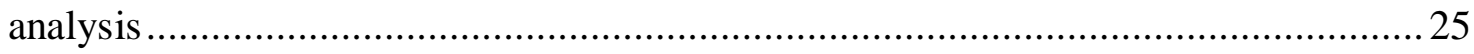

3.3 Depletion of the readily releasable pool and calculating release rates with the

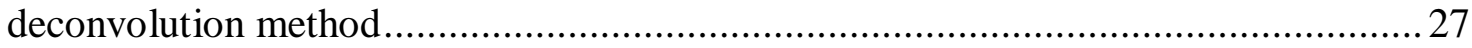

3.4 Saturation of postsynaptic receptors at RS-RS connections in S1 .........................29

3.5 A close look at the blocking effect of Kyn suggests MVR in S1 ...........................34

3.6 S1 connections can induce a postsynaptic AP more reliably than V1 connections. 38 


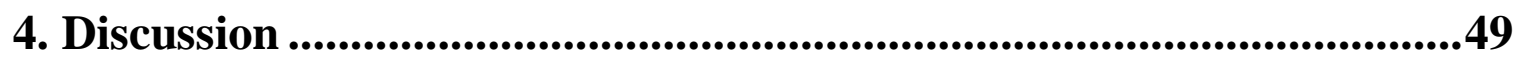

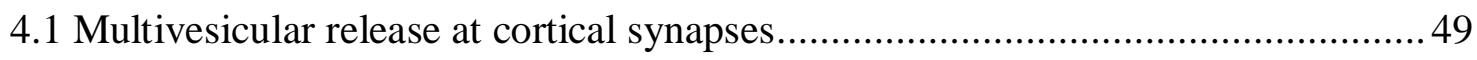

4.2 Differences of synapses at two sensory areas and their physiological implication .51

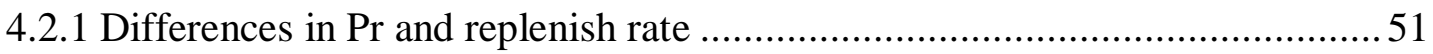

4.2.2 Difference in reliability of synaptic transmission revealed by dynamic clamp 52

4.2.3 Summary of physiological and anatomical data .............................................55

5. Summary ...............................................................................................................58

Bibliography ............................................................................................55

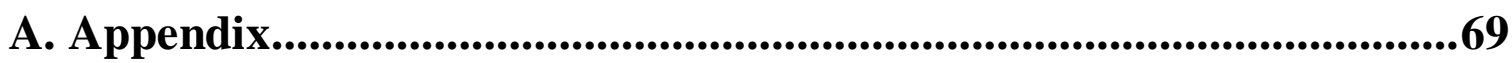

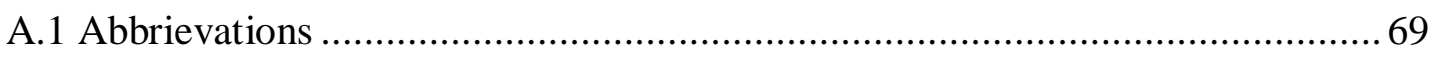

Acknowledgement ...................................................................................70

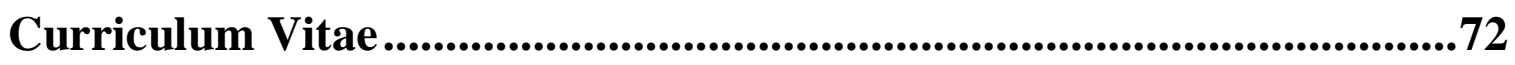

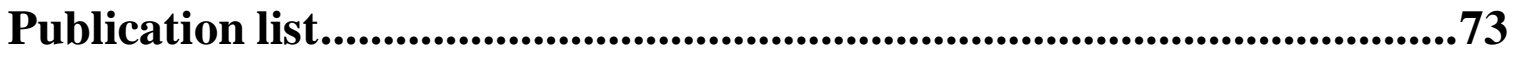




\section{Introduction}

\subsection{Motivation}

"Do we perceive the world passively or do we create the world actively by our brain, and how?" Sensory system plays a key role in our lives; indeed we "define" the world according to how we "sense" it. To understand how sensory systems function hence is a central issue in neuroscience. Various approaches like psychophysics, anatomy, physiology, and molecular biology were taken to solve this issue. Although the question is so big that till now we still don not have a complete answer to it, thanks to all the efforts of neuroscientists, the general anatomical structure of different sensory pathways was established. Among all kinds of senses, the visual system is crucial for higher animals. With the delicate design of the visual neuronal network, we are able to perceive the beauty of the world; therefore I would like to understand the circuitry within the visual system. Much is known about the general neuroanatomy of the visual sensory system by now. It has been long established that the visual sensory system begins at the retina. Light is received here and then the information is transmitted through the optic nerve and thalamus and finally to the visual cortex.

\subsection{Top-down, system approaches to the visual function}

The visual cortex can be split up into different areas: the primary visual cortex (V1), V2 area, V3 area, V4 area, and V5 area, and they represent different stages of visual information processing (Kuffler S. et al., 1984). Among them, the best studied area is V1 because it is structurally well-defined and is also the earliest cortical visual region which 
is essential for the most basic visual information processing. In V1, information first arrives in layer 4 (L4), then goes on to layer $2 / 3$ (L2/3), and finally is sent out to the higher cortex or thalamus through layer 5 (L5) or 6 (Ferster and Lindstrom, 1983). In addition to the anatomical findings, a special feature of the sensory neurons in the visual pathway called a receptive field has been found physiologically. Extensive research has defined the receptive field of a neuron, which is a spatial (and temporal in some cases) pattern in which the stimuli excite or inhibit the firing of the neuron, and this defines the selectivity of the neurons to particular sensory inputs. The receptive fields can be different in size and shape. In the retinal ganglion cells and the thalamic relay neurons, the receptive field is circular with a concentric On/Off center/surrounding whereas cortical neurons have elongated receptive field, which has a specific orientation preference (Hubel and Wiesel, 1962). The neurons with certain elongated receptive field respond the best to a bar with special orientation instead of a spot in the receptive field like the neurons of the retinal ganglion and the thalamic relay neurons.

The differences in the receptive field properties have been an area of extensive research. It has been hypothesized that the differences in the receptive field properties reflect the processing of visual information. More importantly, it is postulated that the orientation selectivity makes us be able to "see" the outline of an object which is the precondition for V1 to perform one of its tasks, detecting luminance contrast (see above). Furthermore, a distinctive structure, the orientation selective column, has been identified in V1. This column intersects through the six-layer structure of the cortex, and the neurons in each column share the receptive field with similar orientation selectivity. The signal of the preferred orientation is transmitted mainly within the column (Hubel and Wiesel, 1962) which indicates that a well-defined circuitry exists to perform a specialized function which is the orientation selectivity in V1.

Various models have been suggested how the orientation selectivity emerged. Hubel and Wiesel (Hubel and Wiesel) postulated that orientation selectivity emerges in the thalamocortical synapses. They argued that the convergence of thalamo-cortical synapses to a cortical neuron is sufficient to form an elongated receptive field. However 
in contrast to the Hubert and Wiesel model, Creutzfeldt and colleagues (Pei et al., 1994) have proposed that the intra-cortical connection plays a major role in orientation selectivity, although the importance of the thalamocortical synapses cannot be neglected. These models have been around for over 40 years, but there is still no general consensus on how orientation selectivity emerges. Also a detailed explanation of the network on cellular level is lacking due to the resolution of the techniques such as extracellular recordings in vivo and functional magnetic resonance imaging which were used in previous researches. Nevertheless, despite the differences in the models it has been agreed that, L4 neurons, as the first gate for signals to get into the cortex, play an important role in filtering the visual signal and the formation of elongated receptive field.

\subsection{Bottom-up, cellular approaches to the visual function}

To compensate for the top-down approach, neuroscientists have come from the other end of the spectrum - a bottom-up approach was taken. Anatomical classification of cell types and connection patterns has been characterized in cat visual cortex (Ahmed et al., 1994; Binzegger et al., 2004; Gilbert, 1983; Gilbert and Wiesel, 1979). Physiologically, the neurons in each layer of neocortex were classified according to their firing patterns upon a current injection. Majorly three classes were described. Regular spiking neurons (RS) show adapting trains of spikes, and each spike has a relatively long half-width with complex afterhyperpolarization and afterdepolarization. Fast-spiking neurons (FS) fire at a high frequency with no adaptation, and the spikes they generate are brief with fast, deep monophasic afterhyperpolarization. The third type is intrinsically bursting neuron. This type of neurons has a tendency to generate a burst responding to a just-threshold stimulus. Despite the burst, an individual spike of intrinsically bursting neurons is quite similar to that of RS neurons (Connors and Gutnick, 1990). Gupta A. et al. further expanded these three types into nine classes. Firstly, neurons were broadly classified as nonaccommodating, accommodating, and stuttering cells. These three major classes were then subdivided into three sub-groups according to the characteristics of the onset of the response (Gupta et al., 2000). In principle, pyramidal cells/ spiny stellate cells belong to the RS neurons (or non-accommodating neurons) which form excitatory synapses, and 
other interneurons belong to the rest of firing types which form inhibitory synapses. Furthermore, descriptive synaptic features such as short-term depression/facilitation were also documented (Beierlein et al., 2000, 2003; Gupta et al.). For example, RS to FS connection is a depressing synapse whereas RS to low-threshold spiking neurons (LTS, another subtype classified by Beierlein M. et al., 2000 which should belong to the accommodating class) is a facilitating synapse (Beierlein et al.). Moreover, molecular identity of different classes of neurons was also documented (Monyer and Markram, 2004). However, there is a huge gap between the knowledge at the cellular level and the functional system level. The logic of synaptic connections and the mechanism underlying the neural circuitry still remain unclear in visual cortex. If we take the whole visual pathway as a big machine which processes the raw materials (light stimulation) into the final product (images we perceive), knowing how the cables are wired (anatomy) and which possible computational units (cell type and descriptive features) exist is not enough to explain how the machine produce the product. Without knowing how each computational unit operates upon different inputs (synaptic transmission mechanism) and the current weight of each cables carries (synaptic strength and synaptic plasticity), it is impossible to deconvolve the whole producing process into the circuit diagram. Therefore, research on the mechanism of synaptic transmission and synaptic plasticity is essential to fill the gap.

\subsection{From a cell to a synapse}

\subsubsection{Fluctuation analysis}

Synaptic plasticity is a collative term of all kinds of modifications in synaptic strength depending on neuronal activities. According to the time scale of such changes, it can be further categorized into long-term and short-term plasticity. Long-term plasticity refers to those modifications which last longer than tens of minutes to hours whereas short-term plasticity happens within the range of milliseconds (ms) to seconds (s). My research will focus on the latter part because the time scale of incoming sensory signals is similar to that of short-term plasticity (Cook et al., 2003; Zucker and Regehr, 2002). 
More importantly, short-term plasticity makes a synapse play an active role in real-time information processing with a strong computational power (Abbott and Regehr, 2004; Abbott et al., 1997).

Synaptic strength is determined by both pre- and post-synaptic factors. Del Castillo and Katz established the quantum theory by careful observations of synaptic responses at the neuromuscular junction synapse. They found that the amplitude histogram plot of end-plate potentials recorded from the muscle showed a poisson/binomial distribution with several peaks. More importantly, these peaks are separated with equal distance which indicates that a synaptic response composes of at least one quantum, and the distribution of quantum amplitude is a normal distribution with the same mean value. A larger synaptic response is a sum up of several quanta. This beautiful property of synaptic response resulted in the key concept of quantum theory: the synaptic strength is set by three parameters, the number of releasable units $(\mathrm{N})$, release probability $(\operatorname{Pr})$, and the quantal size $(\mathrm{q})$. These parameters in turn determine the physical constrains of synaptic plasticity (Zucker, 1973). It is worthwhile to note that the definition of $\mathrm{N}$ at Katz's time was just an physically ambiguous term, "quantal units". Nowadays its physical identity is still controversial. It can refer to the number of independent release sites, the number of releasable vesicles, or morphologically defined number of active zones. The Pr is the probability that synaptic vesicles can be released. The q represents the synaptic response evoked by only one vesicle (one quantum). According to the definitions, a synaptic response can be formulated as below:

\section{Post-synaptic current=N* $\mathrm{p} * \mathrm{q}$}

Following the quantum theory, a binomial model was applied to the amplitude histogram plot of the post-synaptic responses to estimate these parameters in different preparations (Buhl et al., 1997; Gulyas et al., 1993; Korn et al., 1981). It was reported that the number of quantal peaks in the histogram plot matches with the number of anatomical synaptic contacts, which led the authors to postulate the "one site, one vesicle" concept (see below and Discussion). However, this method can be applied only at those synapses in which 
the amplitude histogram plot displays clear peaks, and this condition can only be achieved when $\mathrm{N}$ and/or $\mathrm{Pr}$ is relatively small. Therefore, fluctuation analysis was introduced so that the analysis can be applied more generally (Redman S.J., 1990; Faber D.S. and Korn H., 1991; Silver R.A. et al., 1998; Clements J.D. and Silver R.A., 2000; Scheuss V. and Neher E., 2001). Initial attempts of fluctuation analysis were done by analyzing the coefficient of variation (c.v.) of post-synaptic responses to distinguish the source of synaptic plasticity and to estimate the three quantal parameters $(\mathrm{N}, \operatorname{Pr}$ and $\mathrm{q}$, Faber D.S. and Korn H., 1991; Feldmeyer D. et al., 1999; Feldmeyer D. et al., 2002). For the latter purpose c.v. analysis was not ideal because one of the three synaptic parameters has to be known or assumed in order to obtain the other two. Silver et al. hence developed variance-mean (V-M) analysis (multiple probability fluctuation analysis) which does not need strong assumptions to estimate the $\mathrm{N}, \mathrm{Pr}$, and $\mathrm{q}$ from synaptic responses. The variance and mean are calculated from the fluctuation of synaptic responses responding to one action potential (AP). An essential feature of this method is that it explores the synaptic response fluctuations at different $\operatorname{Pr}$ (altering $\left[\mathrm{Ca}^{2+}\right]$ ), and because of multiple points in variance-mean plot it provides more information about the underlying synaptic mechanisms. Here, the $\mathrm{N}$ was defined as the number of independent release sites. Assuming the vesicle release follows a binomial model, the V-M plot of synaptic response fluctuations at different Pr settings displays a parabola relationship. From the V-M plot, one can estimate the N, Pr, and q. In addition, because the V-M analysis is based on the binomial model which assumes q does not change with time or under different conditions, the relationship of V-M plot would be distorted if this assumption is violated,. This property potentially helps us to detect the changes in $\mathrm{q}$ (for example, the change at the post-synaptic site due to post-synaptic receptor desensitization or saturation). Scheuss V. and Neher E. further extended to apply V-M analysis to the synaptic responses during a train of action potentials (Scheuss V, and Neher E., 2001). Instead changing the Pr by for example altering $\left[\mathrm{Ca}^{2+}\right]$, this method samples from the dynamic Pr responding to a train of APs. This way the experimental protocol is simplified because there is no need of measuring under various conditions. Therefore it can be potentially used more widely (Biro et al., 2005; Scheuss et al.; Taschenberger et al., 2002). 
The V-M analysis was used in different preparations to gain a mechanistic insight of synaptic plasticity (Silver et al., 1998; Scheuss V. et al. 2002; Taschenberger H. et al., 2002; Silver et al., 2003; Biro A. et al, 2005). However, not much has been done in the cortical synapses due to the complexity of neuronal types and the lack of detailed knowledge of fine cortical anatomy. Nevertheless, the most investigated cortical region is the somatosensory cortex (S1). It is also called Barrel cortex for the distinct feature of barrel shaped structures in L4, and the feature has in fact made S1 the most popular region for cortical/sensory research. Each whisker is represented somatotopically in the large-scale anatomical structure of a barrel in L4 of the neocortex (Woolsey and Van der Loos, 1970). The barrel structure is formed by clustered L4 neurons which receive inputs from the same whisker (Armstrong-James and Fox, 1987; Simons, 1978; Welker, 1976). This structure can be recognized easily in the acute brain slice preparation under light microscopy (Agmon and Connors, 1991). These characters therefore allow us to relate the physiological function in vivo to the neuronal circuitry at the level of individual neurons and synaptic connections in vitro. In S1, the synaptic parameters have been estimated in L4-L4 excitatory connections using c.v. analysis (assuming $\mathrm{N}$ is the number of morphological synaptic contacts) (Feldmeyer et al., 1999) and L4-L2/3 excitatory connections by V-M analysis (Silver et al., 2003). The latter one is the first application of $\mathrm{V}-\mathrm{M}$ analysis to the cortical neurons. It was reported that the number of functional release sites was equal to the number of morphological synapses in this type of connection. Furthermore, it was suggested that each synaptic contact released a single vesicle (independent of Pr) and that the intrinsic Pr was high, so this type of synapse must be tuned to response reliably to spatially distributed, timing-based signals. In addition to L4-L2/3 synapses, the synaptic transmission between L4-L4 neurons was also reported to be reliable (Feldmeyer et al., 1999). However, it is obvious that different sensory cortex may have different anatomical characters. In cat visual cortex, clear structure of orientation columns and "blobs" can be seen, but in rat or mouse there is no defined column structure like Barrel cortex in the visual cortex. The logic of how neurons are wired must be different, but does this difference also exist at the synaptic level? What would be the basic universal unit in the brain, a cell or a synapse? What is the smallest 
unit that makes our brain able to perform different tasks? Taking the same type of synapse in S1 as a reference, we explored the mechanism of synaptic transmission in V1 connections by V-M analysis with train stimulation in our research and tried to get closer to the answers of these questions mentioned above.

\subsection{2 “one-site, one-vesicle” hypothesis v.s. multivesicular release}

Another line of mechanistic insight rooted in the quantum theory is the manner in which synaptic vesicles are released upon a single nerve impulse. As mentioned above, the definition of $\mathrm{N}$ was originally the number of releasable units which did not refer to any specific entity with a physical meaning. It could be the number of synaptic contacts, the number of readily-releasable synaptic vesicles, or the number of functional release sites. Based on different physical interpretations of $\mathrm{N}$ orgininally defined by Katz and his colleagues, two distinct hypotheses of the release process were proposed. The first hypothesis, so-called "one-site, one-vesicle" suggests that only one vesicle can be released at one synapse upon one AP (Biro et al., 2005; Buhl et al., 1997; Egger et al., 1999; Feldmeyer et al., 1999; Gulyas et al., 1993; Korn et al., 1981; Murphy et al., 2004; Silver et al., 2003). Korn H. et al. did simultaneous recordings and histological reconstruction on inhibitory synapses at the goldfish Mauthner cell. It was shown that there is only one active zone at one terminal bouton, and the binomial $\mathrm{N}$ matches with the number of bouton identified by the reconstruction; therefore the idea that the site number, $\mathrm{N}$, refers to the number of anatomically defined synaptic contacts was proposed. More evidence of the binomial $\mathrm{N}$ matching with anatomical synapse number was reported to support the idea (Biro et al., 2005; Buhl et al., 1997; Egger et al., 1999; Feldmeyer et al., 1999; Gulyas et al., 1993; Murphy et al., 2004; Silver et al., 2003). Since the number of synapses follows the binomial model, only one vesicle can be released at one AP from one synapse. Based on this model, changes in synaptic strength at one synapse are all or none, and thus reduced the computation power of a single synapse. In addition, the rate of vesicular replenishment following a release event becomes crucial for a steady-state rate of neurotransmission (Dobrunz and Stevens, 1997; Stevens and Wang, 1995). Furthermore, following this hypothesis, a mechanism explaining the "single vesicular 
constraint" was proposed by Dobrunz et al. in 1997. They observed that after one vesicle is released, the second one cannot be released for a short period (tens of ms), and they suggested that this is how only one vesicle can be released by one AP at one synapse.

On the other hand, an alternative hypothesis is multivesicular release (MVR) which provides more flexibility by allowing variation in the number of vesicles released by an AP (Li et al., 2009; Oertner et al., 2002; Tong and Jahr, 1994; Wadiche and Jahr, 2001; Watanabe et al., 2005). Tong and Jahr first reported that the glutamate concentration in the synaptic cleft changes at different Pr. If there is only one vesicle released at one synapse, the glutamate concentration should be the same regardless of Pr. Therefore, they suggested that indeed one synapse can release more than one vesicle which results in a different interpretation of binomial $\mathrm{N}$. The $\mathrm{N}$ refers to the number of functional release sites instead of the number of synaptic contacts. The phenomenon of change in glutamate concentration at synaptic cleft was also reported in several different preparations with different methods (Li et al., 2009; Oertner et al., 2002; Wadiche and Jahr, 2001; Watanabe et al., 2005). According to this hypothesis, a synapse can actually function as an analog device whereas the "one-site, one-vesicle" hypothesis predicts a single synapse as a digital device, and as a result, multiple synapses are required to show graded modulation of signals. More computation power is given to a synapse with MVR. In addition, MVR reduced the failure rate of vesicle release at one synapse at the same Pr. Considering the condition of $\mathrm{N}=3$ and $\mathrm{Pr}=0.6, \mathrm{MVR}$ would predict a failure rate of (1$0.6) 3=0.064$ at one synapse (assuming all three sites locate at one synapse) whereas the "one-site, one-vesicle" hypothesis predicts a failure rate of $(1-0.6)=0.4$ at one synapse. Moreover, asynapse with MVR suffers more from the saturation/desensitization of postsynaptic receptors which further reduces the fluctuation of synaptic strength (Foster et al., 2002; Harrison and Jahr, 2003; Trussell et al., 1993). This does not happen with "onesite, one-vesicle" hypothesis because no matter the post-synaptic receptors are saturated/desensitized or not, the c.v. is the same, and the fluctuations at a synapse level are all or none. Combining lower failure rates and modulations of post-synaptic receptors, MVR makes a synapse more reliable. Taken together, it is important to identify the manner of vesicle release because it leads to totally different interpretation of a 
computational unit of a synapse and different prediction of synaptic reliability. As mentioned before, MVR was reported in several preparations such as hippocampal neurons (Oertner et al., 2002; Tong and Jahr, 1994), climbing fiber-Purkinji neuron synapses (Wadiche and Jahr, 2001), inner hair cell synapse (Li et al., 2009), and Calyx of Held (Taschenberger et al., 2002). However, the existence of MVR, particularly at small cortical synapses, remains controversial (Feldmeyer et al., 1999; Silver et al., 2003).

\subsection{Aim of this study}

In this study, we determined the quantal properties of excitatory connections betweenRS neurons in L4 of V1 and S1 quantitatively. This connection is known as a model system of cortical synaptic transmission as previously described (Feldmeyer et al., 1999; Petersen, 2002; Stern et al., 1992). These neurons in L4 receive signals from the thalamus and further transmit them to other layers in the cortex. At the same time, synaptic interactions between RS neurons often connect to each other within a short distance which allows better clamp for electrophysiological approaches (Lefort et al., 2009; Lubke et al., 2000; Petersen and Sakmann, 2000). In this study we estimated basic properties of small cortical synapses in two major cortical regions. By comparison of these properties, we addressed the issue of synaptic homo/heterogeneity in the cortex. Furthermore, dynamic clamp experiments revealed the importance of such heterogeneity at synaptic level to the reliability of synaptic transmission. 


\section{Material \& Methods}

\subsection{Slice preparation}

Coronal slices $(300 \mu \mathrm{m})$ were prepared from the visual cortex of P22-P28 NMRI mice with a vibrating microtome (VT1000/S1200S; Leica, Wezlar, Germany) (Fig. 1). We started to collect slices at one slice prior to when the hippocampus showed up (approximately $1000 \mu \mathrm{m}$ from the rear end of the cortex). In total 3-4 slices were collected for following recordings. 


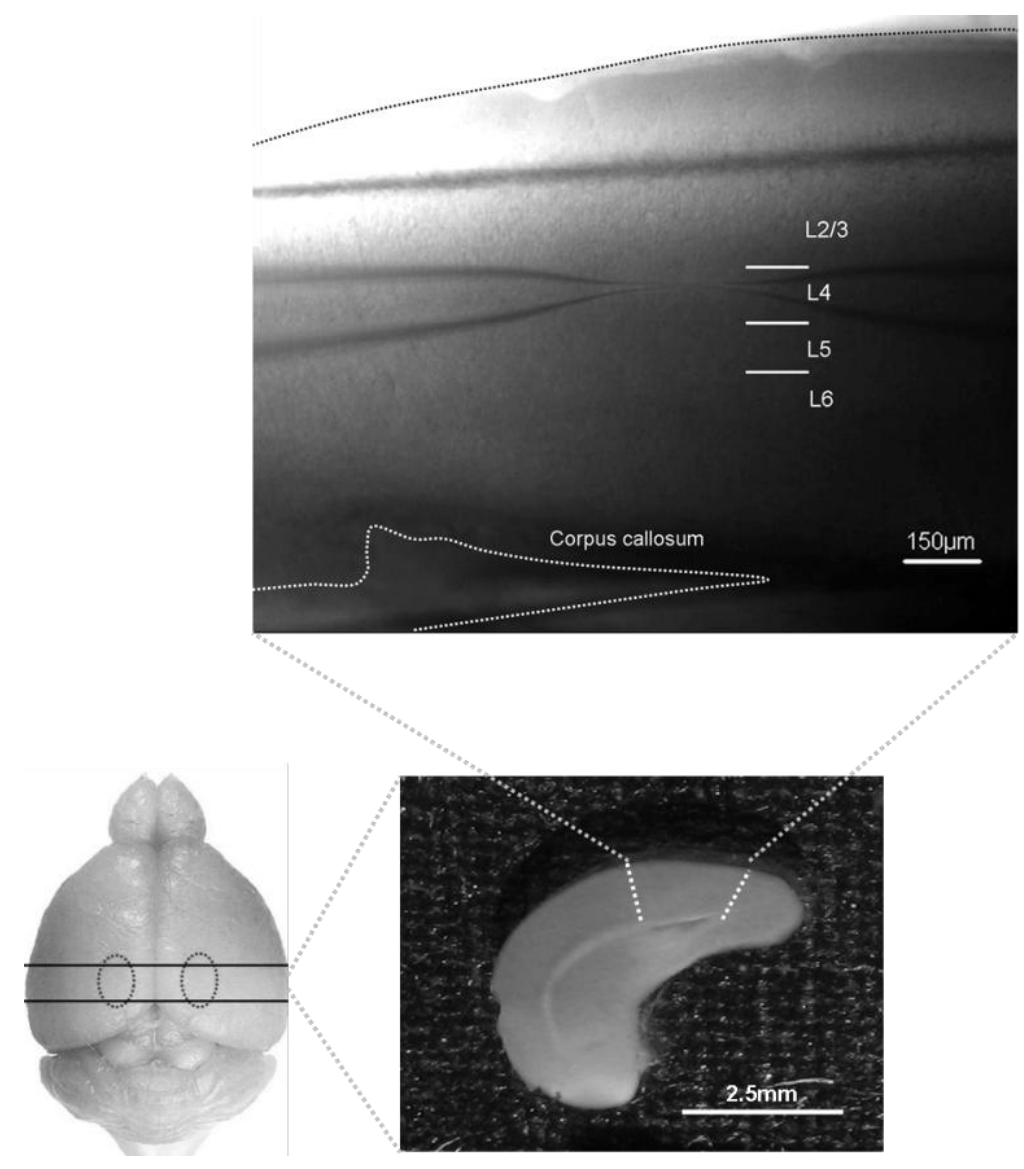

Figure 1 Coronal slice from mouse visual cortex

Region where slices were dissected is located between the two black solid lines of the brain

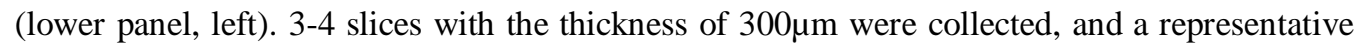
slice is shown here (lower panel, right). Visual cortex locates in between the two white dotted lines, and the view with simultaneous paired recording pipettes under the $4 \mathrm{x}$ objective is shown in the upper panel. Though the boundary of each layer is not necessarily clear, L4 neurons can be identified according to the cell morphology and the relative location to other layers (see below electrophysiology).

For somatosensory cortex, thalamocortical brain slices $(300 \mu \mathrm{m})$ were prepared from P19-P24 mice according to Agmon A. and Connors B.W.,1991 (Fig. 2). Different from coronal sections of the visual cortex, a $55^{\circ}$ angle relative to the mid-line of two hemispheres was taken to preserve the thalamocortical fibers. These fibers are the landmark of the start of collecting slices for recording. At least 6 slices could be 
collected. If the cutting angle is correct, the barrel structure should be visualized clearly, and recordings were done on the RS neurons within barrels.
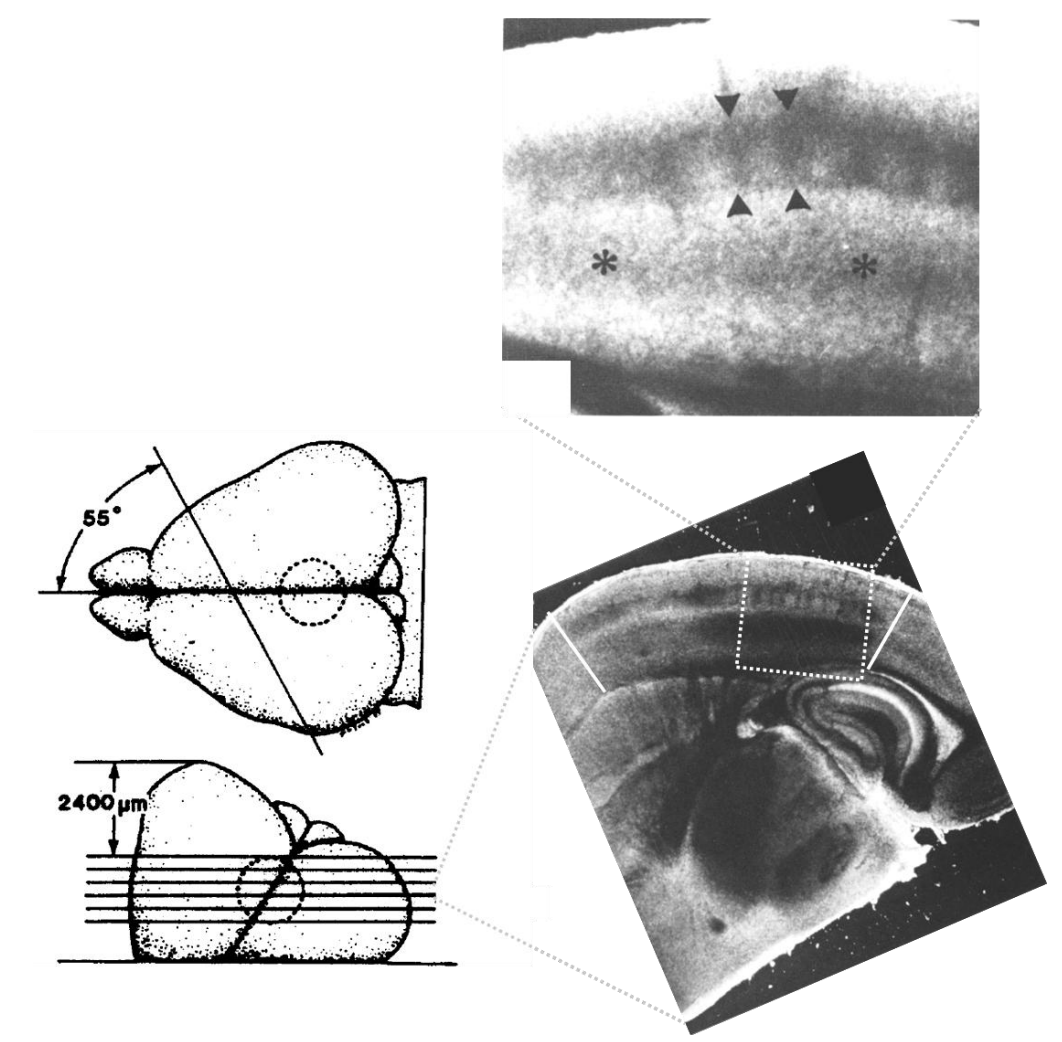

Figure 2 Thalamocortical slice from mouse somatosensory cortex (adapted from Agmon and Connors, 1991)

The cutting angle and the region where slices were dissected and collected are shown in the

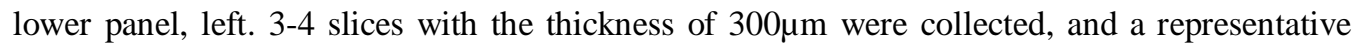
slice is shown here (lower panel, right). S1 cortex locates in between the two white dotted lines, and the barrel structure can be seen clearly even with naked eyes. The upper panel shows the enlarged view of the square region in the lower panel, left. Cells located in the barrels were chosen for our recordings.

The slicing solution contained the followings (in $\mathrm{mM}$ ): $125 \mathrm{NaCl}, 2.5 \mathrm{KCl}, 25$ $\mathrm{NaHCO}_{3}, 1.25 \mathrm{NaH}_{2} \mathrm{PO}_{4}, 3 \mathrm{MgCl}_{2}, 0.1 \mathrm{CaCl}_{2}, 0.4$ L-ascorbic acid, 3 myo-inositol, 2 pyruvate, and 25 glucose. Before slicing, the slicing solution was frozen till half ice and half liquid, and to be bubbled with $95 \% \mathrm{O}_{2} / 5 \% \mathrm{CO}_{2}$. For animals older than P26 for visual cortex and for those older than P23 for somatosensory cortex, a sucrose solution was used for slicing instead of normal slicing solution. The sucrose solution contained 
(in $\mathrm{mM}$ ) $60 \mathrm{NaCl}, 2.5 \mathrm{KCl}, 120$ sucrose, $25 \mathrm{NaHCO}_{3}, 1.25 \mathrm{NaH}_{2} \mathrm{PO}_{4}, 3 \mathrm{MgCl}_{2}, 0.1 \mathrm{CaCl}_{2}$, 0.4 L-ascorbic acid, 3 myo-inositol, 2 pyruvate, and 25 glucose. After a brain slice was cut, it was transferred to a chamber with extracellular solution, and was incubated at $37^{\circ} \mathrm{C}$ for an hour. During the incubation, the solution was always bubbled with $95 \% \mathrm{O}_{2} / 5 \% \mathrm{CO}_{2}$. The extracellular solution contained (in $\mathrm{mM}$ ) $125 \mathrm{NaCl}, 2.5 \mathrm{KCl}, 25 \mathrm{NaHCO}_{3}, 1.25$ $\mathrm{NaH}_{2} \mathrm{PO}_{4}, 1 \mathrm{MgCl}_{2}, 2 \mathrm{CaCl}_{2}, 0.4 \mathrm{~L}$-ascorbic acid, 3 myo-inositol, 2 pyruvate, and 25 glucose. After incubation, the slices were stored in the same solution at room temperature and was bubbled with $95 \% \mathrm{O}_{2} / 5 \% \mathrm{CO}_{2}$.

\subsection{Electrophysiology}

L4 neurons can be recognized by the round shape and the small size of the somata (Feldmeyer et al., 1999; Stern et al., 1992). Also, the relative location of those neurons in the six-layer structure of the cortex was carefully examined. For visual cortex, there is no clear boundary in between each layer, but the neurons in different layers show distinct morphological characters, so one can still tell in which layer the recordings were done. From the outer most edge of a slice, the molecular layer is firstly encountered. No cells located in this layer. With the direction to corpus callosum, the next layer to molecular layer is L2/3 where the neurons are pyramidal shaped with medium size surrounded by interneurons. And then the next layer is L4 where the neurons have round shape and relatively small size. L5 comes next to L4 and L5 neurons are distinct due to the large size and clear pyramidal shape. The last layer, layer 6, is characterized by the small round shaped neurons with lots of fibers running over the region. Normally, we looked for L5 neurons first because they are easy to be recognized. After finding L5, we moved toward the molecular layer until the small, round L4 neurons were seen. To further confirm they are indeed located in L4, we moved further toward the molecular layer until the characters of L2/3 neurons were found. L4 neurons should be located in between L2/3 and L5. Whole-cell voltage clamp recordings were performed on the soma of L4 neurons. After a connected pair was identified, excitatory post-synaptic currents (EPSCs) under different conditions were recorded (EPC 10, HEKA, Lambrecht, Germany) at a sampling rate of $50 \mathrm{kHz}$ (filtered at $3-6 \mathrm{kHz}$ ). The resistance of glass pipettes we used 
was 3-5 MOhm, and the intracellular solution contained (in $\mathrm{mM}$ ) 140 potassium gluconate, $20 \mathrm{KCl}, 10 \mathrm{HEPES}, 5$ ATP-Mg, 5 Phosphocreatine, 0.5 GTP, and 0.2 EGTA and was adjusted to $\mathrm{pH} 7.2$ with $\mathrm{KOH}$. The osmolarity of this solution was around 330 mOsm. Liquid junction potential (around $10 \mathrm{mV}$ ) was not corrected. Series resistances were all below $20 \mathrm{MOhm}$, and 20-50\% compensation was used. The data were further off-line filtered with a low pass filter at $1 \mathrm{kHz}$ before analysis. In some experiments, 50 $\mu \mathrm{M}$ D(-)-2-amino-5-phosphonopentanoic acid (D-AP5, Tocris) was applied, but Nmethyl-D-aspartic acid receptors (NMDARs) did not contribute to the peak EPSC amplitudes in our study (Fig.3). To prevent saturation of postsynaptic $\alpha$-Amino-3hydroxy-5-methyl-4-isoxazolepropionic acid receptors (AMPARs), $0.5 \mathrm{mM}$ kynurenic acid (Kyn, Tocris) was added to extracellular solution in some experiments. In addition, in some experiments $10 \mathrm{mM}$ tetraethylammonium (TEA, Sigma) was applied to increase the releasing probability. In RRP depletion experiments, a half or two third of the potassium gluconate was replaced with cesium gluconate and 10mM TEA was also applied to block potassium channels, and to prevent repolarization. Also, D-AP5 was always applied in this experiment. All experiments (except experiments in Fig.5 and Fig.6) were performed at $30-35^{\circ} \mathrm{C}$. 
A
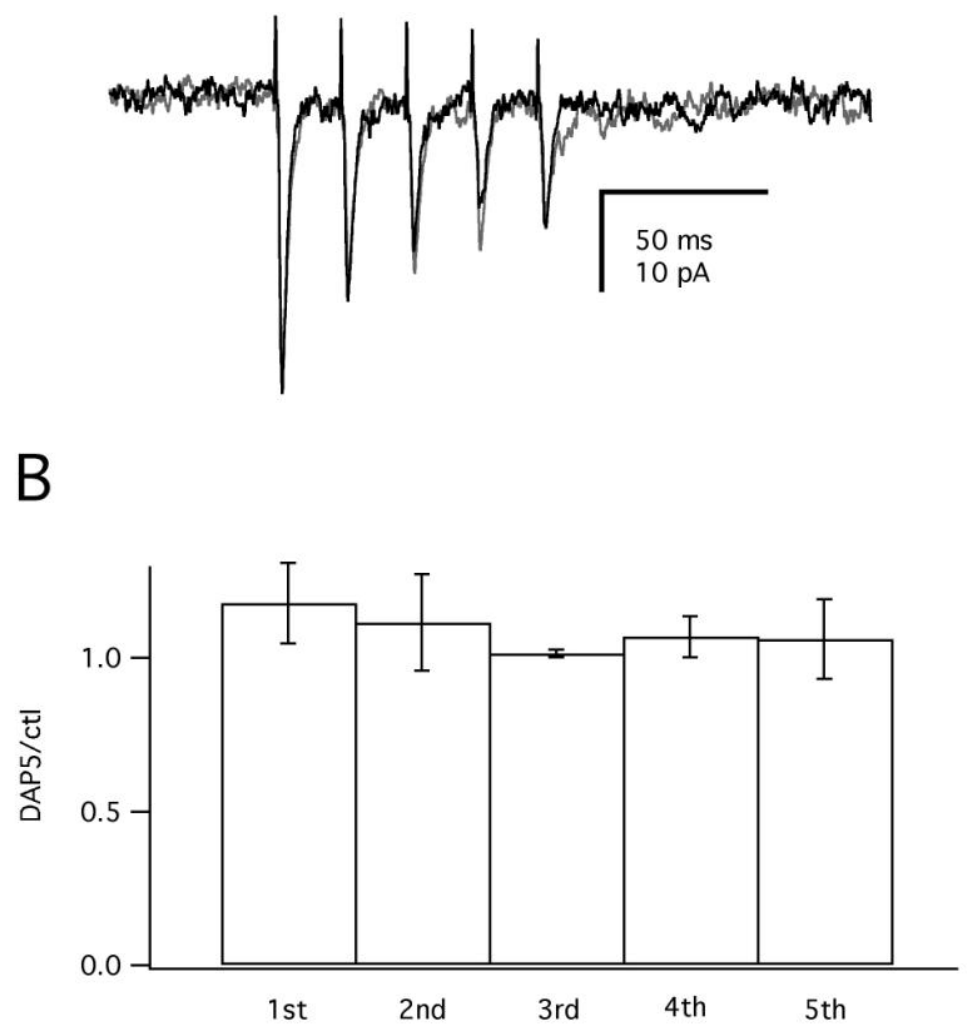

Figure 3 The effect of DAP5

At S1, it has been reported that NMDA receptor-mediated EPSCs were prominent at negative potential (Feldmeyer et al., 1999; Stern et al., 1992). We examined this issue in our experimental condition (more matured animals and under physiological temperature) at L4 synapses in S1. Under voltage clamp, control traces (grey) and the traces under DAP-5 (black) were averaged over 10 times and are displayed in A. In B, the ratio of the EPSC amplitudes (DAP5/Ctl) is displayed from the $1^{\text {st }}$ to the $5^{\text {th }}$ EPSC during a stimulus train. The values range between 1 and 1.2, indicating that NMDA receptors do not contribute to the peak EPSC amplitudes in our experiments.

\subsection{Variance-mean analysis}

$50 \mathrm{~Hz}$ train stimulations were applied to a pre-synaptic neuron and EPSCs were recorded from a post-synaptic one. The stimulus train was applied every $10-20 \mathrm{sec}$ and was repeated more than 20 times. The data were excluded from analysis when rundown 
of the EPSCs was noticed. The amplitude of each EPSC was calculated from the average of five data points around the peak subtracting the baseline of each peak. The baseline was the average over 50 points $(1 \mathrm{~ms})$ just before the onset of EPSCs. The stimulation was repeated for more than 20 times and the amplitude of each peak EPSC was taken for analysis. We averaged the EPSCs of each peak (the $1^{\text {st }}, 2^{\text {nd }}, 3^{\text {rd }}$ and so on) over all stimuli and the mean and the variance were obtained. These two parameters of each peak were plotted against each other. The estimation of N, Pr, and q was done according to Scheuss and Neher (2001). Definition of $\mathrm{N}$ is the number of functional release sites or vesicle number of readily releasable pool (RRP) (assuming $\mathrm{P}_{\text {occupancy }}=1$ ) which should be a fixed number. Pr refers to the combination of probability of vesicle occupancy at the slot and vesicular release probability, and $\mathrm{q}$ is the postsynaptic current amplitudes induced by a single vesicle release (Verejone.D, 1966). Based on the binomial model, the variance and mean plot of EPSCs is predicted to follow a parabola relationship which can be derived as follows:

$$
\operatorname{Mean}_{\mathrm{EPSC}}=\mathrm{N} * \operatorname{Pr} * \mathrm{q},
$$

and

$$
\text { Variance }_{\mathrm{EPSC}}=\mathrm{N} * \operatorname{Pr} *(1-\mathrm{Pr}) * \mathrm{q}^{2}
$$

If we combine the two equations above, we would obtain:

$$
\begin{aligned}
& \mathrm{y}=(1 / \mathrm{N}) * \mathrm{x}^{2}+\mathrm{q} * \mathrm{x} \\
& \text { where } \mathrm{y} \text { : Variance } \mathrm{EPSC}_{\mathrm{EPS}} ; \mathrm{x} \text { : Mean } \text { EPSC }_{\mathrm{EPC}}
\end{aligned}
$$

Figure 4 illustrates how the parabola relationship of variance and mean develops with different Pr. When Pr is low, only the initial part of the parabola is plotted, so instead of a parabola, the V-M plot shows a linear relationship. When Pr is higher than 0.5 , a parabola relationship can be observed. 


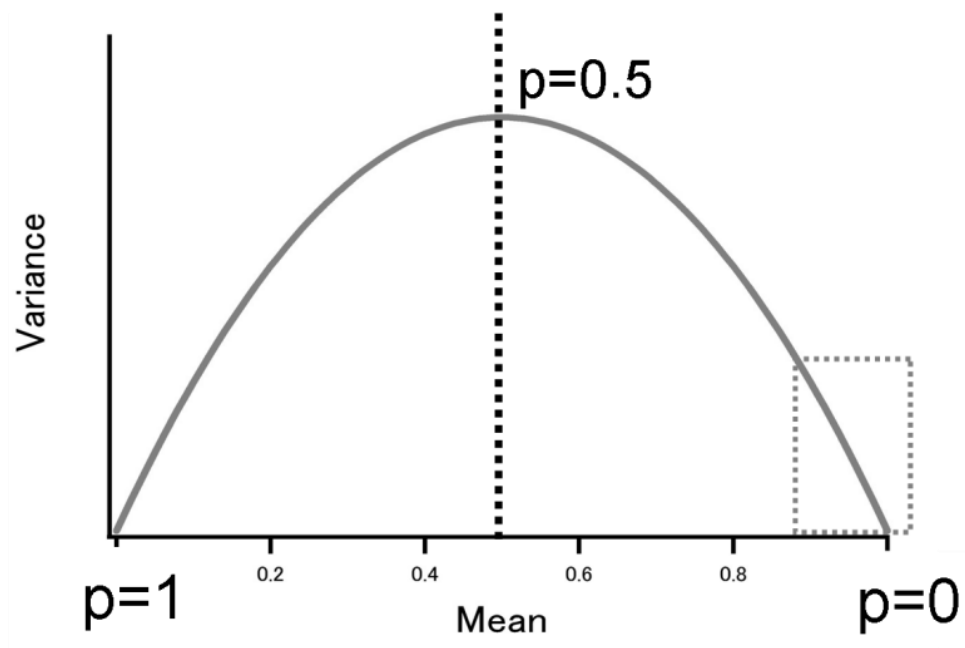

Figure 4 Illustration of varianc- mean relationship

Fitting the data points of variance mean plot with a parabola, the $\mathrm{q}$ and $\mathrm{N}$ can be estimated. The initial slope of the parabola is $\mathrm{q}$ which can be derived as follows:

$$
y^{\prime}=(2 / N) * x+q
$$

When $\mathrm{x} \rightarrow>0$,

$$
y^{\prime}=q
$$

From the intercept of the parabola with the $\mathrm{x}$-axis $\mathrm{N}$ can be calculated.

$$
\mathrm{y}=0, \mathrm{x}=0 \text { or } \mathrm{N} * \mathrm{q}
$$

Therefore,

$$
\mathrm{N}=\mathrm{x} / \mathrm{q}(\text { when } \mathrm{y}=0)
$$

When $\mathrm{N}$ is known, the Pr can be further calculated by dividing the first EPSC by the product of $\mathrm{N}$ and $\mathrm{q}$.

$$
\operatorname{Pr}=1^{\text {st }} \operatorname{EPSC} /(\mathrm{N} * \mathrm{q})
$$

Note that other sources of fluctuation make the analysis more complicated (see results) .

\subsection{Deconvolution}

The EPSCs were deconvolved with the miniature EPSC (mEPSC) to estimate transmitter release rates (Diamond and Jahr, 1995; Van der Kloot, 1988). In practice, we used the same procedure as Neher and Sakaba (2001), except that no residual current component due to delayed clearance of glutamate was assumed. In each cell pair, the 
decay time constant of mEPSC was adjusted by varying the time constant until spontaneous events become delta-pulse like events in the release rate trace. The mEPSC amplitudes were assumed to be $5 \mathrm{pA}$ in the presence of $0.5 \mathrm{mM}$ Kyn, which was verified by variance-mean analysis (see below, Results).

\subsection{Dynamic clamp experiments}

The simulated excitatory synaptic current was injected to the soma by a home-built hardware realization of dynamic clamp setup. It composed of three stages: input signal filtering and conditioning (analog circuit), digitalization (Analog Devices AD 7495) and calculation (Atmel AT32AP7000), digital to analog conversion (Analog Devices DA5620). The control program was written in $\mathrm{C}$ (with development environment AVR32 Studio 2.5.0 and compiler avr32-gec 4.3.2). The simulated synaptic current I was calculated from the voltage-independent conductance $\mathrm{g}$ and the instantaneous driving force, $\mathrm{V}-\mathrm{E}$.

$$
\mathrm{I}=\mathrm{g} *(\mathrm{~V}-\mathrm{E})
$$

$\mathrm{V}$ is the membrane potential measured from patch clamp amplifier and fed to the dynamic clamp in each cycle. Here the I-V relationship is assumed to be linear. $\mathrm{E}$ is the reversal potential for AMPA receptors, which is set to $0 \mathrm{mV}$. $\mathrm{g}$ is modeled by a double exponential waveform

$$
\mathrm{g}=\mathrm{G} *\left(\text { - exp }\left(-\mathrm{t} / \mathrm{tau} \_ \text {rise }\right)+\exp \left(-\mathrm{t} / \mathrm{tau} \_ \text {decay }\right)\right) \text {. }
$$

$\mathrm{G}$ is a scaling factor for the peak amplitude. Tau_rise and tau_decay were the time constants for the rising and decaying phase of the waveform and they are taken from fit to the recorded EPSCs. In each cycle of the dynamic clamp experiment, an updated I was calculated according to (1) and (2) and fed to the soma through the patch pipette. For each simulated synaptic event, the peak amplitude of the conductance was a positive random number from a Gaussian distribution with the calculated mean and S.D. from recorded EPSC peak amplitudes (Table 2). The update rate of dynamic clamp was 50 $\mathrm{kHz}$. Experiments were done in the presence of $10-25 \mu \mathrm{M}$ bicuculine (Sigma). 


\section{Results}

\subsection{Variance-mean analysis and the mEPSC amplitude measurement at L4 neurons in V1}

We performed simultaneous paired or triple recordings on the somata L4 RS neurons in mouse visual cortex. First, the neuronal type was identified according to the firing pattern of a neuron upon a current injection. These L4 neurons were then classified into three categories: RS neuron, FS neuron, and LTS neuron (Fig. 5) (Beierlein et al., 2003; Buhl et al., 1997; Gupta et al., 2000). While RS neurons form excitatory synapses onto the postsynaptic neuron, FS and LTS neurons form inhibitory synapses. To test whether a pair of neurons was synaptically connected, we depolarized one of the two neurons from $-80 \mathrm{mV}$ to $0 \mathrm{mV}$ for $2 \mathrm{~ms}$ to induce an action potential and examined if we could record mono-synaptic, time-locked responses from the other neuron, and vice versa. Furthermore, to exclude di-synaptic connections, only those connections were selected for analysis in which the time difference between the onset of EPSCs and stimulation of the pre-synaptic neuron was restricted to less than $5 \mathrm{~ms}$. In this study, we focused on only RS-RS connections in L4. 

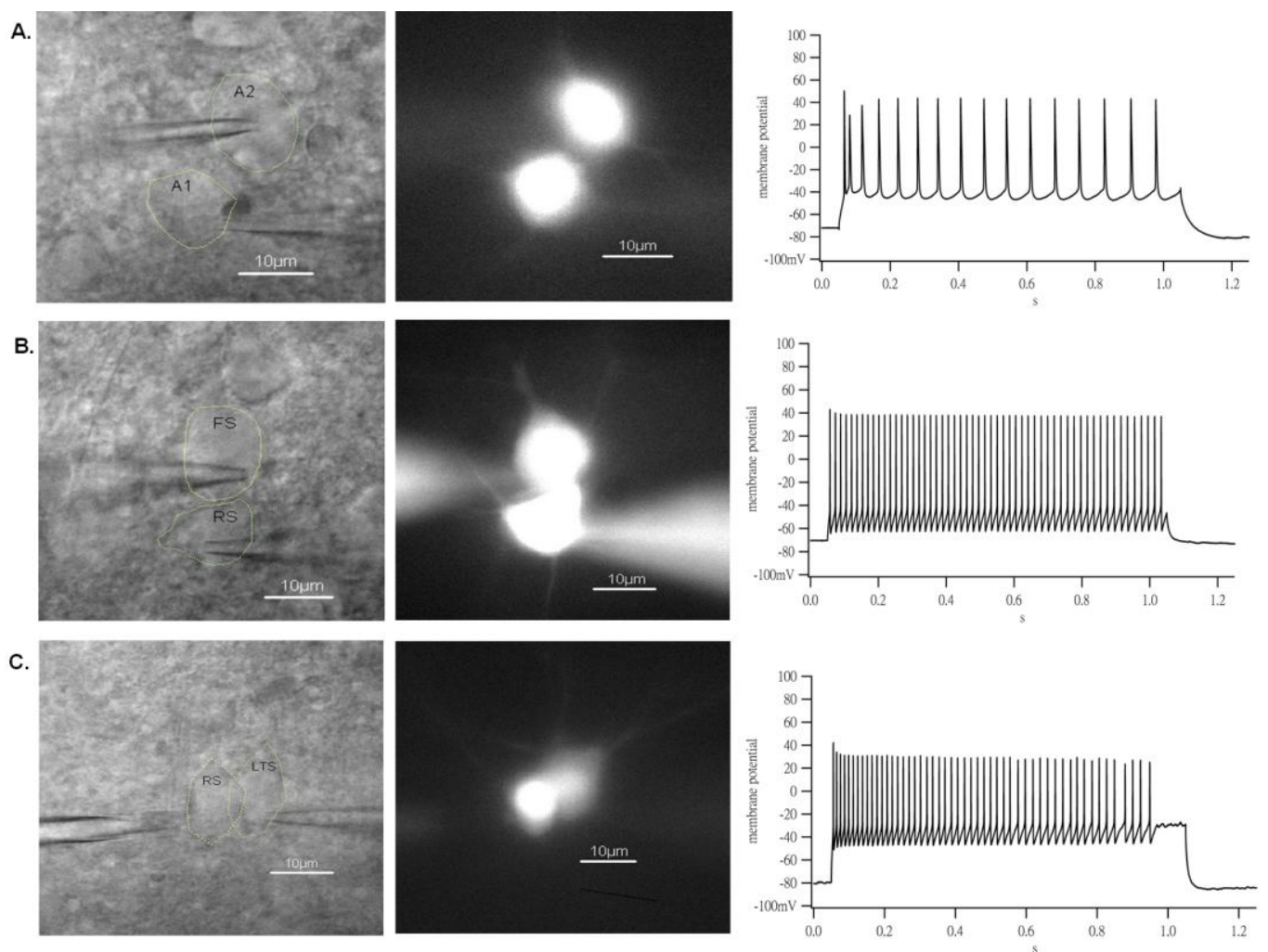

Figure 5 Three classes of excitatory connections in L4 of V1

The configuration of a paired recording from two neurons under bright field, and fluorescence images are shown (left, and middle). The right panel shows the firing pattern of the postsynaptic neuron. (A) The connection between two RS neurons. The right panel shows the firing pattern of a RS neuron. (B) the connection between a RS neuron and a FS neuron. The right panel shows the firing pattern of a FS neuron. (C) the connection between a low-threshold spiking neuron and a LTS neuron. The right panel shows the firing pattern of a LTS neuron.

After a connected pair was identified, $50 \mathrm{~Hz}$ train stimulations (depolarizations from $-80 \mathrm{mV}$ to $0 \mathrm{mV}$ for $2 \mathrm{~ms}$, to induce an action potential at the soma, which spreads to the terminal) were applied to the presynaptic neuron and the EPSCs were recorded from the post-synaptic one. Figure 6A illustrates pre- and post-synaptic currents elicited by such stimulation under $2 \mathrm{mM}$ extracellular $\mathrm{Ca}^{2+}$ at room temperature from a representative connection in V1. A train-stimulation allowed us to sample EPSCs at different releasing probabilities (Scheuss V. and Neher E., 2001) within one trace. By repeating this train stimulation with an interval of $10 \mathrm{~s}$ more than 20 times, variance and mean of the EPSC amplitudes of each stimulus were obtained, and variance-mean analysis was applied to determine the synaptic parameters: N, Pr, and q (See Material and methods). Figure 6A 
shows that the relationship between variance and mean was linear. This indicates that $\operatorname{Pr}$ is low, and only q could be obtained from the analysis. On average, the q estimated by variance-mean analysis was $9.2 \pm 2.9 \mathrm{pA}(\mathrm{n}=3$, before correcting for the c.v. of the mEPSCs of $27 \%$, see below).

In addition to variance-mean analysis, we looked into the individual traces of each connection. As shown in figure $6 \mathrm{~B}$, after the $9^{\text {th }}$ stimulus the EPSCs started to fluctuate in an all-or-none manner, and the amplitudes of the success events were the same, so it most likely resulted from a single vesicle release. On the contrary, the $5^{\text {th }}$ EPSC showed a variety of EPSC amplitude, indicating more than one vesicle was released from the terminal at the $5^{\text {th }}$ stimulus. We examined those EPSCs after the $9^{\text {th }}$ stimulus in those three connections, and 49 events were observed. The failure rate was higher than 0.5 at each stimulus (when we pooled all stimulus, the overall failure rate was higher than 0.9), and the average amplitude of successful events was $9.3 \pm 0.7 \mathrm{pA}(\mathrm{n}=49)$. The amplitude here matched with the result of variance-mean analysis, implying that the estimates by our method were valid.

To further confirm our results, we measured the evoked mEPSCs and constructed the mEPSC histogram. For measuring the evoked mEPSCs, the extracellular $\mathrm{Ca}^{2+}$ was reduced to 0.4-0.6 mM (Isaacson and Walmsley, 1995; Katz and Miledi, 1965), and the divalent cation concentration was maintained by increasing the concentration of $\mathrm{Mg}^{2+}$ to $3 \mathrm{mM}$. A $2 \mathrm{~ms}$ depolarization (from $-80 \mathrm{mV}$ to $0 \mathrm{mV}$ ) was applied repetitively to the presynaptic cell with an interval of $3 \mathrm{~s}$ instead of 10-20 s in this particular set of experiments, and the EPSCs were recorded from the postsynaptic cell. We calculated the failure rate in each connection, and only those ones with a failure rate higher than 0.5 were taken for further analysis. Overall, $126 \mathrm{mEPSC}$ events were recorded from 5 connections, and the average failure rate was 0.6 . The average mEPSC amplitude was $10.8 \pm 0.48 \mathrm{pA}$. The distribution of mEPSC amplitudes were plotted with that of train stimulation experiment in Figure 6C. The histogram of these two methods (evoked mEPSCs and EPSC events during the late period in the train) matched with each other quite well. Also the average 
mEPSC amplitudes were similar and matched closely with quantal amplitudes estimated from variance-mean analysis.

A
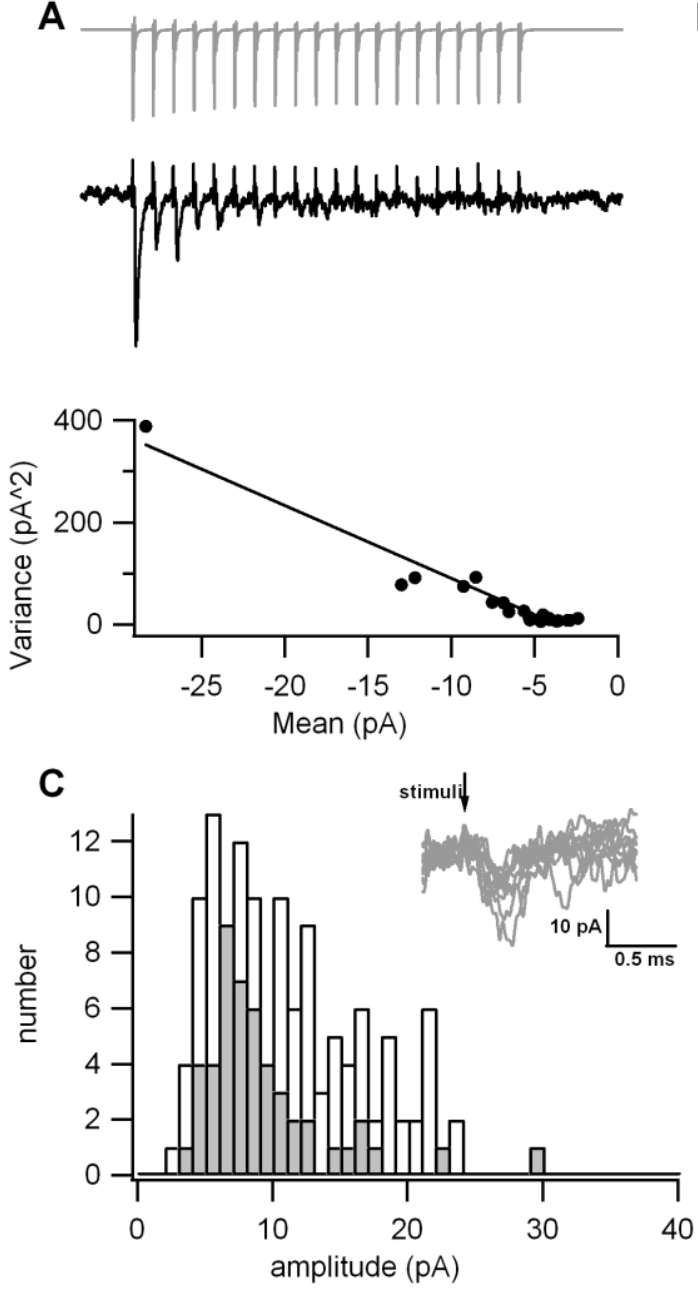

B
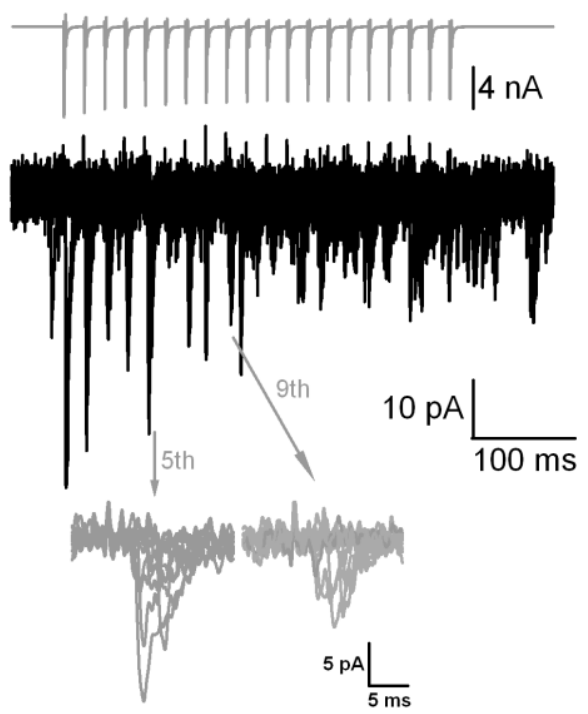

Figure 6 Variance-mean analysis predicts quantal sizes, which matches with the mEPSC amplitudes

(A) The upper panel shows the presynaptic current of a representative RS-RS connection in V1 upon a short depolarization under room temperature, and the middle panel shows the mean EPSC corresponding to the stimuli from the same connection. The variance-mean relationship was plotted in the lower panel. Black solid circles represent the relationship of the variance and the mean of the EPSC amplitudes upon a stimulus train obtained from more than 20 repetitions. The line fit (black line) estimates the $\mathrm{q}=14.2 \mathrm{pA}$. (B) The individual EPSC traces from the same connection as (A) (middle panel). 10 individual traces out of 20 repetitions are superimposed to show the fluctuation of EPSCs. The grey traces are the close look at individual peaks of the 5 th and the 9 th stimuli. (C) The histogram of the evoked mEPSCs 
obatiend under low external $\mathrm{Ca}^{2+}$ (white bar). The inset shows representative mEPSC traces under low $\mathrm{Ca}^{2+}$. Grey bars indicate the histogram of mEPSCs obtained from train stimulation experiments.

The amplitude distribution of mEPSC measurement was slightly skewed to the right, and had a c.v. of 0.52 . From the c.v. of mEPSC distribution, one can estimate the correction factor for q estimated from V-M analysis (see above). The correction factor was introduced here to estimate the maximum error caused by the dispersion of the mEPSC amplitudes, which causes fluctuation of EPSCs and is due to the variability with a given release site (intrasite variability) and among sites (intersite variability). The values of quantal size and binomial parameter $\mathrm{N}$ estimated from $\mathrm{V}-\mathrm{M}$ analysis are denoted as $\mathrm{q}^{*}$ and $\mathrm{N}^{*}$, respectively. These estimates can be subsequently corrected for the variability of mEPSC amplitude distributions, to give corrected quantal size $\mathrm{q}$ and binomial parameter N, according to Silver et al., 1998 as well as Scheuss and Neher, 2001:

$$
\mathrm{q}=\mathrm{q}^{*} /\left(1+\text { c.v. }{ }^{2}\right)
$$

and

$$
\mathrm{N}=\mathrm{N}^{*} \cdot\left(1+\mathrm{W} \cdot \text { c.v. }^{2}\right)
$$

From Fig. 6C, one can calculate the c.v. of mEPSCs, which is 0.52 . The symbol W represents the fraction of quantal variance that is caused by variability between different release sites (Frerking and Wilson, 1996). Because the source of variability in mEPSC amplitude distribution is not exactly known, we tentatively assumed $\mathrm{W}$ to be 0.5 (Meyer et al., 2001). Nevertheless, from c.v. $=0.52$, one can estimate $1+$ c.v. ${ }^{2}=1.27$, suggesting that the actual q might be $27 \%$ smaller (Frerking and Wilson), and $\mathrm{N}$ can be $13 \%$ larger. This error factor can be considered as an upper limit because the mEPSC histogram in Fig.6C may contain the release of more than two vesicles in some cases. If so, c.v. will be significantly smaller. Also, we have not corrected for the effect of jittering of release events (Taschenberger et al., 2005), which is known to cancel out the effect of the mEPSC dispersion. Therefore, the correction factor due to the mEPSC dispersion is likely to be the upper limit. 


\subsection{Estimation of $\mathrm{N}$, Pr, and $\mathrm{q}$ under physiological condition with variance-mean analysis}

To examine the quantal parameters under more physiological conditions, the recording temperature was raised up to $30-32{ }^{\circ} \mathrm{C}$ in the following experiments. We repeated the same $50 \mathrm{~Hz}$ train stimulation protocol on RS-RS connections in V1, and applied variance-mean analysis to the evoked EPSCs in a stimulus train. In Figure 7A (left), the relationship of variance and mean could be fitted with a parabola in some connections. This indicates that temperature raises Pr significantly. From the parabola fit the $\mathrm{q}$ and $\mathrm{N}$ were estimated. The average $\mathrm{q}$ was $9.88 \pm 1.09 \mathrm{pA}(\mathrm{n}=5)$. Possibly, postsynaptic receptor saturation might have caused a parabolic relationship (Foster and Regehr, 2004; Meyer et al., 2001). Therefore, we applied 0.5mM Kyn to the brain slice, and the result is also shown in Figure 7A (black trace in the upper panel; black filled circles in the lower panel). Kyn is a low affinity AMPAR antagonist, so it competes with glutamate but does not block glutamate binding completely when the concentration was not too high. Therefore, it was used to prevent post-synaptic receptors from saturation and desensitization, and to measure the glutamate concentration at the synaptic cleft. An advanced application of this chemical is that one can detect the change in glutamate concentration by observing blocking efficiency under different conditions (Diamond and Jahr, 1997; Wadiche and Jahr, 2001). In this experiment, we mainly used it to protect the receptors from saturation and desensitization so that the assumption of contant quantal sizes in the V-M analysis holds true. With Kyn, the variance-mean relationship of EPSCs was linear in 3 connections (a representative connection is shown in Fig. 7A right) and was parabola in 4 connections (Fig. 7A left) out of 7 connections. Assuming the difference of these two groups (linear v.s. parabola) resulted from the different $\mathrm{Pr}$, the $\mathrm{N}$ could be obtained only from those four with parabola relationship, and the Pr could be calculated only from them as well. The average $N$ was $7 \pm 1.1(n=4)$, and the average $\operatorname{Pr}$ of these 4 connections was $0.59 \pm 0.05(n=4)$. However, the average Pr must be lower than 0.59 because of 3 linear cases $(<<0.5)$. This indicates that the release probability is heterogeneous at V1. 
Though in some cases the Pr was high enough to show a parabolic relationship, the fraction of linear ones in the population was high. Therefore, we attempted to increase the Pr so that we could have more parabola cases, allowing one to estimate $\mathrm{N}$ in all the cases. To achieve this goal, we first tried the conventional way of increasing Pr by elevating the extracellular $\mathrm{Ca}^{2+}$ concentration to 4 or $8 \mathrm{mM} \mathrm{Ca}^{2+}$. However, we could not observe any augmentation of the evoked EPSCs upon high $\mathrm{Ca}^{2+}$ (data not shown). One possible reason could be the surface charge screening effect due to excess divalent cations. Hence, another method was tested, which was to apply $10 \mathrm{mM}$ TEA, a $\mathrm{K}^{+}$ channel blocker, along with $0.5 \mathrm{mM}$ Kyn, a low affinity AMPAR antagonist, in the bath. TEA is known to broaden the action potential waveform by blocking $\mathrm{K}^{+}$channels and increase the $\mathrm{Ca}^{2+}$ flux such that we hoped to increase the Pr. Because rundown of release was faster under TEA (possibly due to the increased Pr), it was difficult to perform variance-mean analysis under both control and in the presence of TEA. Therefore in some experiments, only the TEA condition was tested. The result is shown in Figure 7B. The average Pr was elevated to $0.69 \pm 0.03(\mathrm{n}=11)$, which showed that TEA did increase Pr. However, for a fraction of cells the linear relationship still remained (6 out of 17 connections). We pooled all parabola cases in both experiments (with Kyn only and with Kyn and TEA) assuming that the $\mathrm{N}$ should be intrinsically the same as that of other synapses, and the final estimate of $\mathrm{N}$ was $6.3 \pm 1.0(\mathrm{n}=13)$. 
A
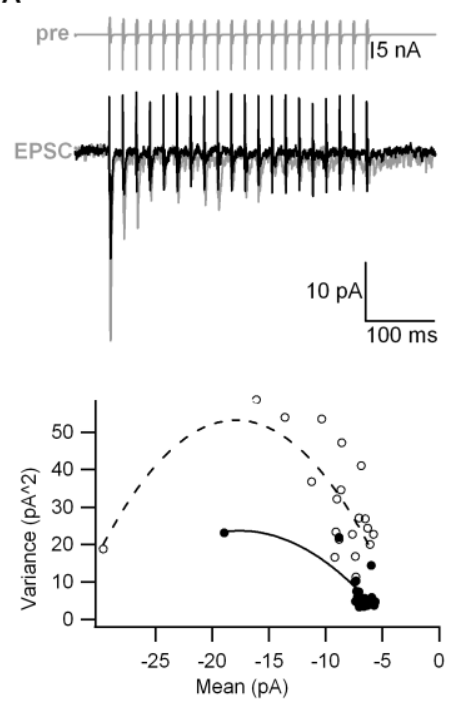

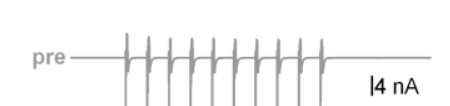

B
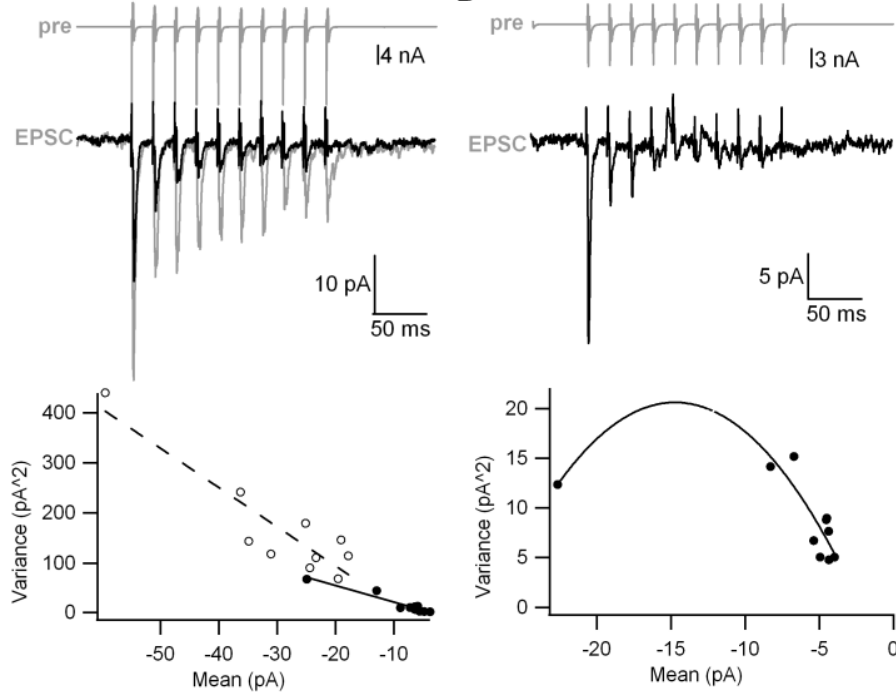

Figure 7 Estimation of $\mathrm{N}, \mathrm{Pr}$, and q in V1 RS-RS connections under physiological condition with variance-mean analysis

The upper panel shows the presynaptic currents of a representative RS-RS connection in V1 upon a short depolarization, and the middle panel shows the mean EPSCs in response to the stimuli from the same connection. The grey trace is the control group, and the black one was obtained under $0.5 \mathrm{mM}$ Kyn. The lower panel shows the variance-mean relationship of the EPSCs both in control (black hollow circles) and in the presence of Kyn (black solid circle). (left) the parabola fit of the control (black dash line) estimates $\mathrm{N}=4.2$ and $\mathrm{q}=8.7 \mathrm{pA}$. The fit of the group under Kyn (black line) estimates $\mathrm{N}=7$ and $\mathrm{q}=5 \mathrm{pA}$. (Right) The linear fit of the control (black dash line) estimates $q=7.8 \mathrm{pA}$. The fit of the group under Kyn (black line) estimates $\mathrm{q}=3.3 \mathrm{pA}$.(B) The order of panels is the same as (A), but the traces are obtained from the experiments with TEA and Kyn in V1. The parabola fit estimates $N=7.6$ and $q=3.9$ pA.

\subsection{Depletion of the readily releasable pool and calculating release rates with the deconvolution method}

In order to verify the results from variance-mean analysis, we performed experiments to deplete the RRP of synaptic vesicles and measured the actual number of released vesicles. Part of the potassium in the intracellular solution was replaced with cesium, a $\mathrm{K}^{+}$channel blocker, and the concentration ratio of $\mathrm{Cs}^{+}$to $\mathrm{K}^{+}$in the intracellular solution was 2 to 1 . We did not replace all $\mathrm{K}^{+}$with $\mathrm{Cs}^{+}$because cells did not tolerate 
such an extreme condition at physiological temperature. After a connected pair was identified, $10 \mathrm{mM}$ TEA was applied to the bath to further block potassium channels and at the same time $0.5 \mathrm{mM}$ Kyn was also added to prevent postsynaptic receptor saturation. With both $\mathrm{Cs}^{+}$and the external TEA, we aimed to have a step-like long depolarization at the terminal, similar to the idea of Katz and Miledi (1967) at the squid giant synapse. As shown in Figure 8 (upper panel), two $100 \mathrm{~ms}$ depolarizations were given to the presynaptic neuron with an interval of $200 \mathrm{~ms}$, and the EPSCs were recorded from the postsynaptic one. The protocol was repeated in one connection several times with an interval of longer than $10 \mathrm{~s}$ for the cell to fully recover from the previous stimulus. The second depolarization was applied to confirm that the RRP was depleted completely, in which case there should be no response during the second pulse. That was the case in 7 cell pairs. While prefect voltage clamp of the presynaptic terminal was not expected, relatively short length of axons (Feldmeyer et al., 1999) together with blocking $\mathrm{K}^{+}$ channels with $\mathrm{Cs}^{+}$might have helped sufficient stimulation of the terminal. The $\mathrm{N}$ was then calculated by the deconvolution method. Figure 8 (lower panel) shows the cumulative trace of release from a representative connection. On average, the $\mathrm{N}$ was 8.3 $\pm 0.9(\mathrm{n}=7)$. There was no significant difference between the results from variancemean analysis and the depletion experiment ( $\mathrm{t}$-test, $\mathrm{p}=0.13$ ). Therefore, we concluded that the estimated $\mathrm{N}$ was valid (the assumption above was fulfilled). The result also suggests that a strong depolarization does not recruit additional pool of vesicles which are not used during an AP. The vesicle replenishment rate following the RRP depletion could be obtained from the cumulative trace. In V1, the time required to refill the whole RRP $\left(\mathrm{T}_{\text {rec }}\right)$ under high $\left[\mathrm{Ca}^{2+}\right]$ was $91.4 \pm 15.5 \mathrm{~ms}(\mathrm{n}=7)$, which was estimated by fitting the cumulative release trace with an exponential (representing the RRP) and a line (representing the replenishment). One may expect to see more recovered response during the second pulse (applied at an $200 \mathrm{~ms}$ interval), given that replenishment rate is less than $100 \mathrm{~ms}$. Possibly, the $\mathrm{Ca}^{2+}$-dependent component of vesicle replenishment is accelerated during the pulse, but drop in $\mathrm{Ca}^{2+}$ after the pulse would decrease the rate of replenishment (Dittman and Regehr, 1998; Hosoi et al., 2007; Wang and Kaczmarek, 1998). 


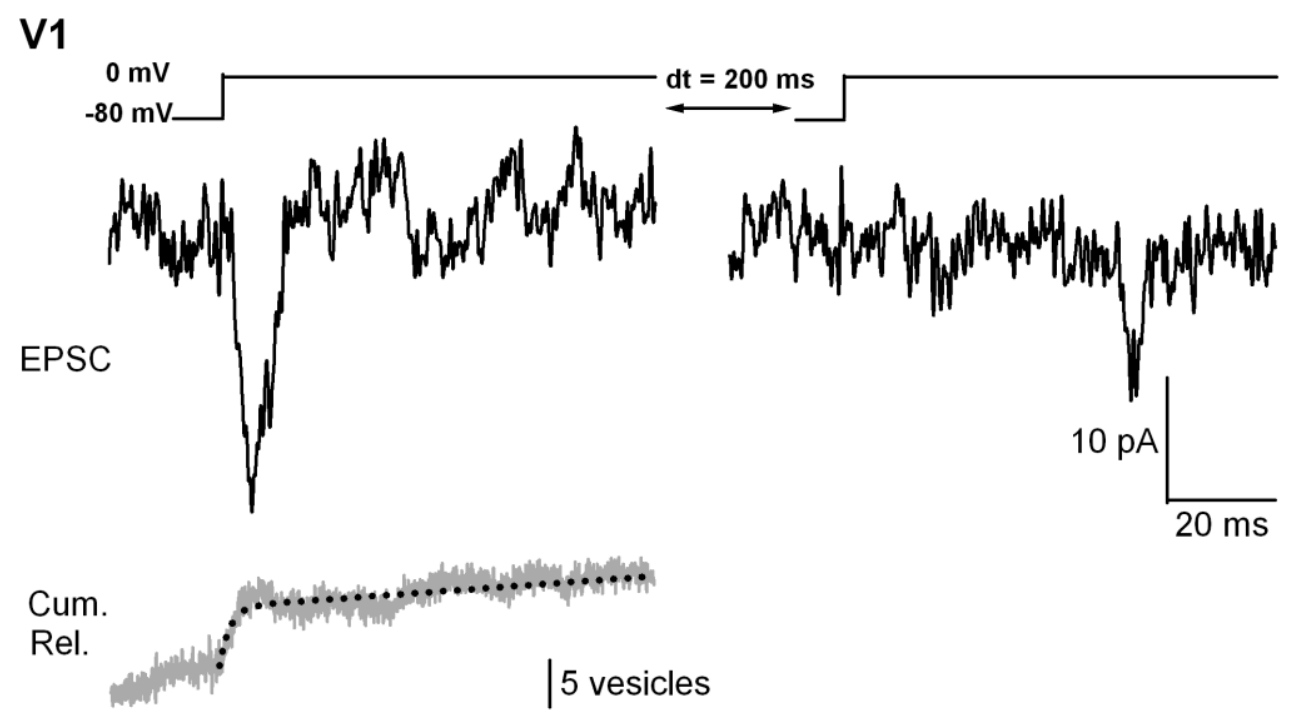

Figure 8 Depletion of the readily releasable pool and calculating cumulative release with deconvolution method in S1 connections

A representative depletion trace from a representative RS-RS connection in V1. The stimulation protocol is shown in the upper panel. The presynaptic neuron was depolarized from $-80 \mathrm{mV}$ to $0 \mathrm{mV}$ for $100 \mathrm{~ms}$, and after an interval of $200 \mathrm{~ms}$, the second $100 \mathrm{~ms}$ depolarization was applied to the cell to test the remaining vesicles within the RRP. One representative individual EPSC responding to a strong depolarization is shown in the middle panel. Upon the second depolarization, there was no vesicle released, indicating depletion of the RRP. The lower panel shows the cumulative trace of release from deconvolution of the EPSC. The two components (an exponential and a line) fit is shown in black dash line. From the fit, the number of vesicle in the RRP was estimated to be 7.4, the time constant of RRP depletion was $0.89 \mathrm{~ms}$, and the vesicle replenishment rate constant was $70 \mathrm{~ms}$ in this case.

\subsection{Saturation of postsynaptic receptors at RS-RS connections in S1}

From the data above, we have obtained the three important quantal parameters $(\mathrm{N}$, Pr, and q) of RS-RS synapses in V1. The N of RS-RS connections in V1 were quite different from those determined previously in S1synapses : According to Feldmeyer et al. (1999), the morphological synaptic contact number was 3-4 (Egger et al., 1999). 
Therefore, we examined whether the synaptic properties were different among different cortical areas. We repeated the variance-mean analysis and the pool depletion experiments at the RS-RS connections in the L4 of S1 at physiological temperature (30$35^{\circ} \mathrm{C}$ ). An example from the variance-mean analysis is shown in Figure 9 (lower panel). In the control group, the variance exhibited a shallow dependence on the mean (black hollow circle). When a parabola was fitted to the data, the intercept of $x$-axis was far above 0 (black dash line). This indicates that the variance-mean analysis is not valid. It is possible that some post-synaptic factors, for example, saturation of the post-synaptic receptors (Foster and Regehr, 2004) distorted the relationship. To test this, we applied $0.5 \mathrm{mM}$ Kyn to the slice and as seen in Figure 9 (lower panel, black solid circle), the variance-mean relationship was restored to parabola shape. Therefore, at RS-RS synapses in S1 post-synaptic receptor saturation played a role during short-term synaptic plasticity, reducing synaptic depression (see below). Under Kyn, the N in S1 was $7.4 \pm$ 1.3 and the $\operatorname{Pr}$ was $0.66 \pm 0.03(n=9)$. There was no significant difference between both values in $\mathrm{S} 1$ and those in $\mathrm{V} 1$ when the data could be fitted with a parabola (t-test, for $\mathrm{N}, \mathrm{p}$ $=0.5$; for Pr, $\mathrm{p}=0.27$ ). It is worthwhile to note that in $\mathrm{S} 1$ all connections showed a parabola relationship under Kyn whereas in V1 about a half of the population was linear. Therefore, the overall Pr in V1 must be lower than S1 and more heterogeneous. 

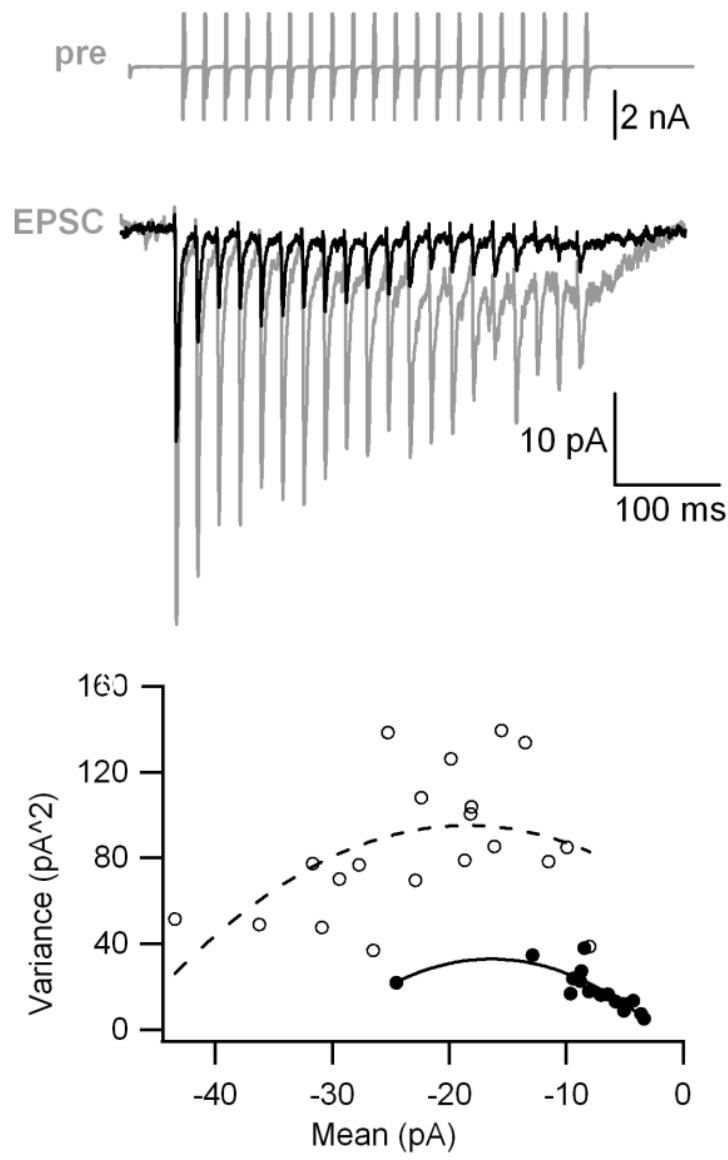

Figure 9 Estimation of $\mathrm{N}, \mathrm{Pr}$, and q q in S1 RS-RS connections under physiological condition with variance-mean analysis

The upper panel shows the presynaptic currents of a representative RS-RS connection in S1 upon a short depolarization, and the middle panel shows the mean EPSCs in response to the stimuli from the same connection. The grey trace is the control group, and the black one was obtained under $0.5 \mathrm{mM}$ Kyn. The lower panel shows the variance-mean relationship of the EPSCs both in control (black hollow circles) and in the presence of Kyn (black solid circle). The parabola fit of the group with Kyn (black filled circles) estimates $\mathrm{N}=6.25$ and $\mathrm{q}=5.3 \mathrm{pA}$.

Next, we performed the RRP depletion experiment to confirm the $\mathrm{N}$ from variancemean analysis. The $\mathrm{N}$ was $8.1 \pm 2.0(\mathrm{n}=7)$, which was not significantly different from the value from variance-mean analysis ( $\mathrm{t}$-test, $\mathrm{p}=0.77$ ). However, this number is much larger than previous estimates of the number of release sites at S1 (3 or 4, Feldmeyer et al., 1998; Buhl et al., 1997; see also Discussion). $\mathrm{T}_{\text {rec }}$ at high $\left[\mathrm{Ca}^{2+}\right]$ in $\mathrm{S} 1$ was $34.3 \pm 6.4$ ms $(n=7)$. The summary of N, Pr, $q$, and $T_{\text {rec }}$ of RS-RS synapses in both cortical areas is shown in Table 1. 


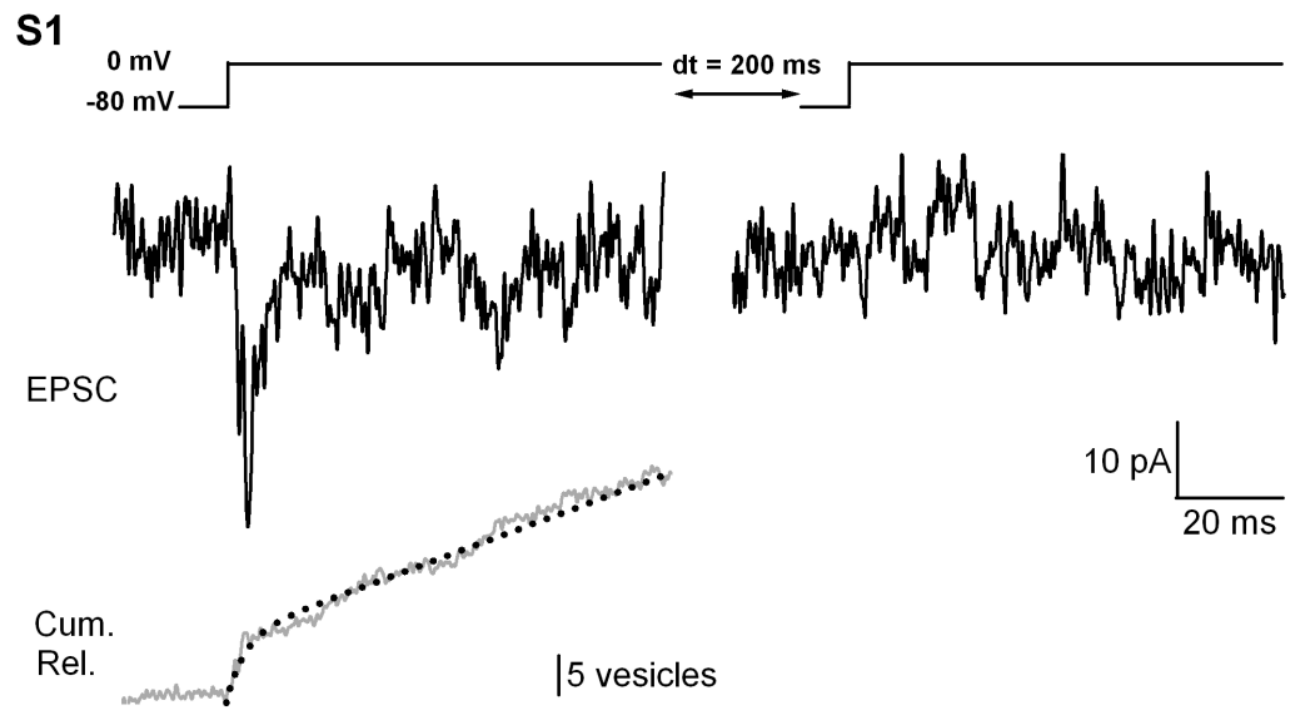

Figure 10 Depletion of the readily releasable pool and calculating cumulative release with deconvolution method in S1 connections

A representative depletion trace from a representative RS-RS connection in S1. The stimulation protocol is shown in the upper panel. The presynaptic neuron was depolarized from $-80 \mathrm{mV}$ to $0 \mathrm{mV}$ for $100 \mathrm{~ms}$, and after an interval of $200 \mathrm{~ms}$, the second $100 \mathrm{~ms}$ depolarization was applied to the cell to test the remaining vesicles within the RRP. One representative individual EPSC responding to a strong depolarization is shown in the middle panel. Upon the second depolarization, there was no vesicle released, indicating depletion of the RRP. The lower panel shows the cumulative trace of release from deconvolution of the EPSC. The two components (an exponential and a line) fit is shown in black dash line. The cumulative trace of release was filtered at $1 \mathrm{kHz}$, so it is less noisy than that in Fig.8, but this would not change the result. The fitting result shows the number of vesicles within the RRP was 7.8 , the time constant of RRP depletion was $3 \mathrm{~ms}$, and the replenishment rate constant was $28 \mathrm{~ms}$ in this example. 


\begin{tabular}{|c|c|c|c|c|c|c|c|}
\hline & C & & 1 & & $\operatorname{Pr}$ & $\mathrm{T}_{\mathrm{re}}$ & \\
\hline & Variance-mean & Mini & Variance-mean & Pool depletion & & Depression curve & Pool depletion \\
\hline V1 & $\begin{array}{l}9.2 \pm 2.9 \mathrm{pA} \\
(\mathrm{n}=3, \mathrm{RT})\end{array}$ & $10.8 \pm 0.48 \mathrm{pA}$ & $\begin{array}{c}7.0 \pm 1.1 \\
(\mathrm{n}=4, \text { w/o TEA }) \\
6.0 \pm 1.3 \\
(\mathrm{n}=11, \mathrm{w} / \mathrm{TEA})\end{array}$ & $\begin{array}{c}8.3 \pm 0.9 \\
(n=7)\end{array}$ & $\begin{array}{c}<0.59 \pm 0.05^{*} \\
(\mathrm{n}=4, \text { w/o TEA }) \\
<0.69 \pm 0.03^{*} \\
(\mathrm{n}=11, \text { w/ TEA })\end{array}$ & $\begin{array}{c}100 \mathrm{~ms} \\
(\mathrm{Pr}=0.45)\end{array}$ & $\begin{array}{c}91.4 \pm 15.5 \mathrm{~ms} \\
(\mathrm{n}=7)\end{array}$ \\
\hline S1 & & & $7.4 \pm 1.3(\mathrm{n}=9)$ & $\begin{array}{c}8.1 \pm 2 \\
(n=7)\end{array}$ & $\begin{array}{c}0.66 \pm 0.03 \\
(n=9)\end{array}$ & $\begin{array}{c}71 \mathrm{~ms} \\
(\operatorname{Pr}=0.55)\end{array}$ & $\begin{array}{c}34.3 \pm 6.4 \mathrm{~ms} \\
(\mathrm{n}=7)\end{array}$ \\
\hline
\end{tabular}

* Considering in V1 some connections didn't show parabola relation in variance-mean analysis, the average Pr should be smaller than the value presented here in the table.

Table 1. Summary of the synaptic parameters under different conditions

Quantal sizes (q), the number of synaptic vesicles within the RRP (N) and release probability are shown. Q was obtained under control conditions, but variance-mean analysis did not work properly at S1. Under Kyn, qunatal sizes were estimated to be $5.5 \pm 0.73 \mathrm{pA}$ and $5.5 \pm 0.7 \mathrm{pA}$ in V1 and S1, respectively. Vesicle replenishment rates were estimated from the cumulative release trace in Fig. 8, 10 or else from the depression curve during a $50 \mathrm{~Hz}$ train under Kyn (Fig. 7, 9). A simple vescile pool depletion model (a single RRP with fixed replensiment rate) could explain the depression curve under kyn, when the Pr was set to 0.55 and 0.45 and the replenishment rate was set to $71 \mathrm{~ms}$ and $100 \mathrm{~ms}$ in S1 and V1, respectively.

In Fig. 10, the sustained EPSCs could be observed following depletion of the RRP. While we assume that this component is mediated by synaptic vesicle replenishment, it is possible that the component is mediated, for example, by delayed clearance of glutamate in the synaptic cleft. In order to verify that it is indeed mediated by ongoing release events, fluctuation analysis was applied. Variance and mean was calculated during the sustained component, which should give a value of q by taking the ratio, multiplied by a factor of 2 (Katz and Miledi, 1972). On average, q of $5.9 \pm 0.6 \mathrm{pA}$ was estimated at S1 (in the presence of $0.5 \mathrm{mM} \mathrm{Kyn}$ ), which was close to the value estimated from variancemean analysis used in Fig. 9. A close match between the two estimates suggests that the sustained component is indeed mediated by release events. Also, there is no compound 
fusion during vesicle replenishment, which means that MVR is mediated by coordinated fusion of synaptic vesicles in response to presynaptic $\mathrm{Ca}^{2+}$ influx. If so, then the quantal sizes would be increased. In V1, the analysis was more difficult to apply, possibly because of lower replenishment rates. Nevertheless, the q was estimated to be $7.8 \pm 0.7$ pA.

\subsection{A close look at the blocking effect of Kyn suggests MVR in S1.}

As shown previously, Kyn restored the distorted variance-mean relationship of RS$\mathrm{RS}$ connections in S1. Because differential block of Kyn is a strong indicator of MVR (Wadiche and Jahr, 2001), we looked into the blocking effect in more detail. Figure 11 illustrates the blocking efficiency of Kyn in both cortical areas. In the S1 group the second and subsequent EPSCs were blocked significantly more than the first one, whereas there is no significant difference in V1. Even in the connections with high Pr, there was no differential block at V1.
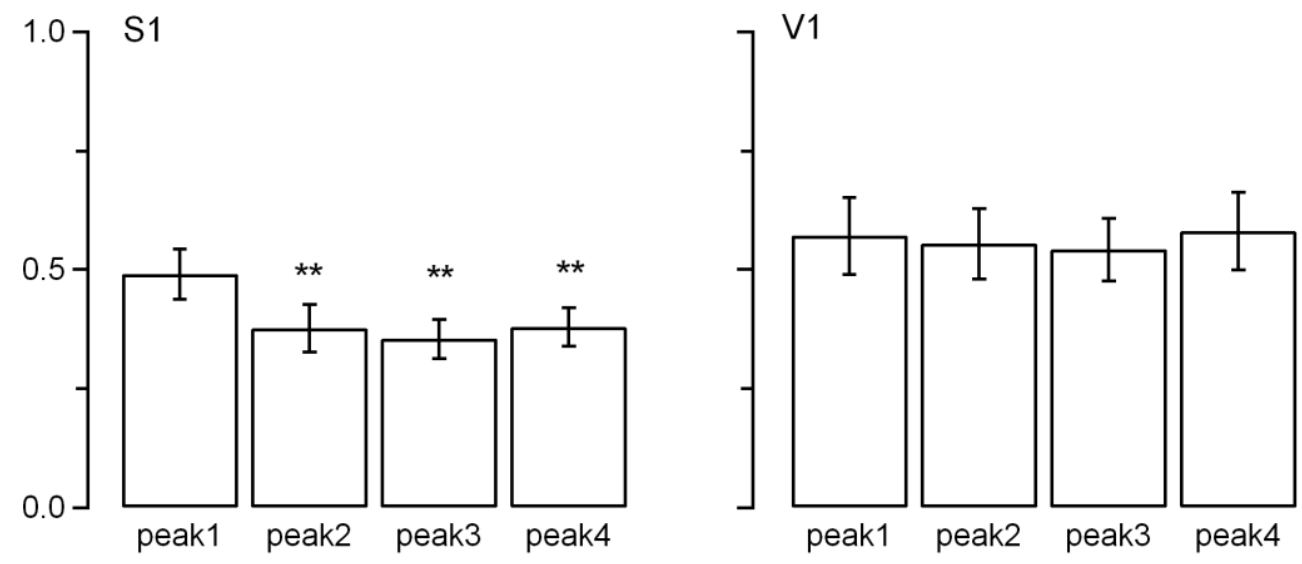

\section{Figure 11 A close look at the blocking effect of $0.5 \mathrm{mM} \mathrm{Kyn}$}

The remaining fraction of EPSC after blocked by Kyn is shown in (A). The EPSCs amplitude of each peak with Kyn were divided by that of the control group. The figure shows the average ratio of the $1^{\text {st }}$ to the $4^{\text {th }}$ peak over all connections we recorded. The left panel shows the results from S1. There is significant difference between the $1^{\text {st }}$ peak and the rest (paired t-test, $1^{\text {st }}$ v.s. $2^{\text {nd }}: p=0.0007 ; 1^{\text {st }}$ v.s. $3^{\text {rd }}: p=0.0009 ; 1^{\text {st }}$ v.s. $4^{\text {th }}: p=0.009$ ). The right panel shows the results 
from V1. There is no significant difference between the $1^{\text {st }}$ peak and the rest (paired t-test, $1^{\text {st }}$ v.s. $2^{\text {nd }}: p=0.81 ; 1^{\text {st }}$ v.s. $3^{\text {rd }}: p=0.72 ; 1^{\text {st }}$ v.s. $4^{\text {th }}: p=0.9$ ).

Differential block was not seen with NBQX (Fig. 12), a high affinity AMPAR antagonist. High affinity (or non competitive) antagonists should decrease the mEPSCs equally. If the differential block was still seen, the mechanisms other than MVR, most likley a voltage clamp problem must be involved (larger EPSCs have more clamp errors) (Wadiche and Jahr, 2001). Taken together, the results rather suggest that the local glutamate transient in the synaptic cleft changes during the train stimulation in active synapses in S1 but not in V1 under physiological condition.

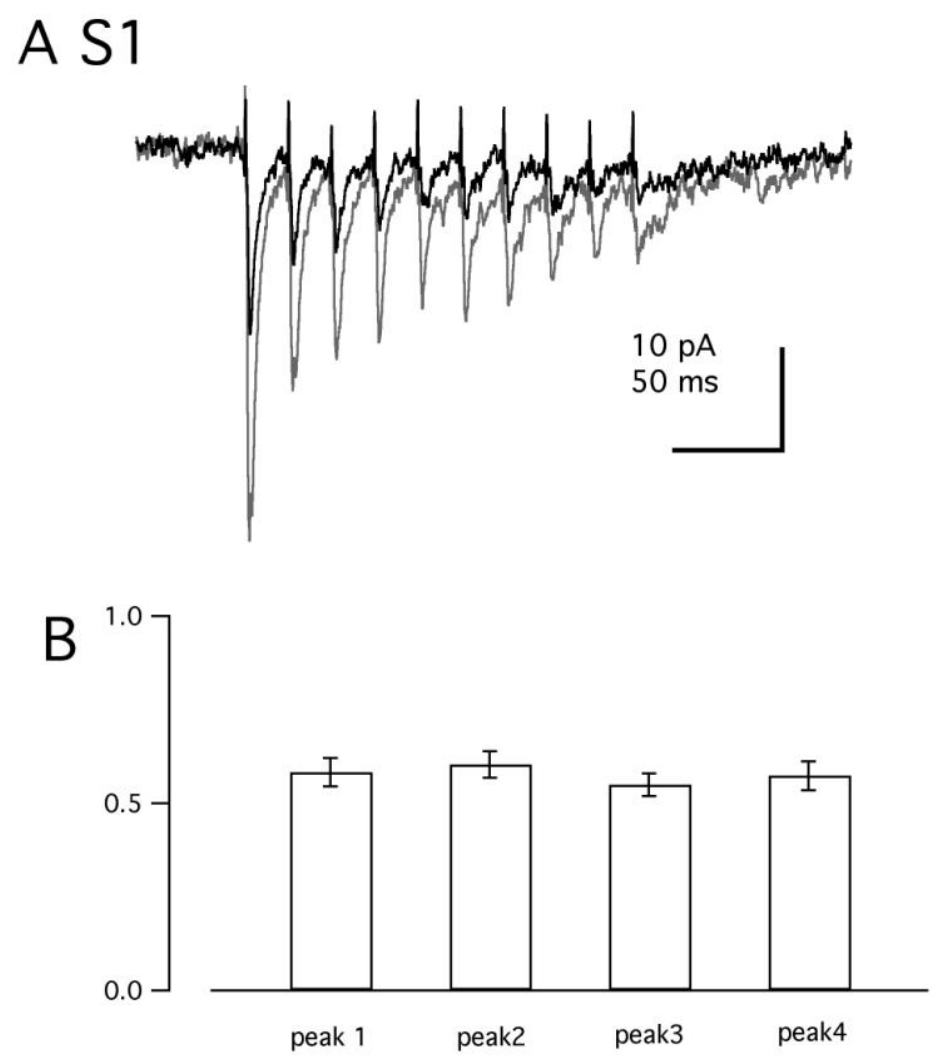

Figure 12 The blocking effect of NBQX on the EPSCs at S1.

Similar to Fig 11, but a low concentration of NBQX (100 - $200 \mathrm{nM})$ was applied to the connected pair of L4 neurons in S1, to examine the blocking efficiency during a train stimulation. Because NBQX is a high affinity AMPA receptor antagonist, all the EPSCs would be blocked equally even in the presence of MVR. The Panel A shows a typical example showing the average traces under control condition (grey trace) and the condition in the 
presence of NBQX (black trace). B shows the summary plot of blocking efficiency, illustrating that the first four EPSCs during a stimulus train were blocked equally by NBQX ( $\mathrm{n}=6$ cell pairs, statistically insignificant $\mathrm{p}>0.05)$. The result also indicates that the improvement of voltage clamp under the receptor antagonist is not the reason for differential block by Kyn.

However, when TEA was applied to further increase Pr, a differential block of Kyn was also observed in V1 (Fig. 13). Though the effect is still less than in S1 under control condition, these data suggest that MVR could occur at RS-RS connections in V1 under high Pr, but predominantly only one or no vesicle is released at a given site in the majority of the connections in V1 under physiological condition. Because MVR happens at elevated Pr, the main reason for a single vesicle release under control condition must be primarily due to a low Pr rather than some special transmitter release mechanism which limits a single vesicle release, such as single vesicle constraint or lateral inhibition of release.

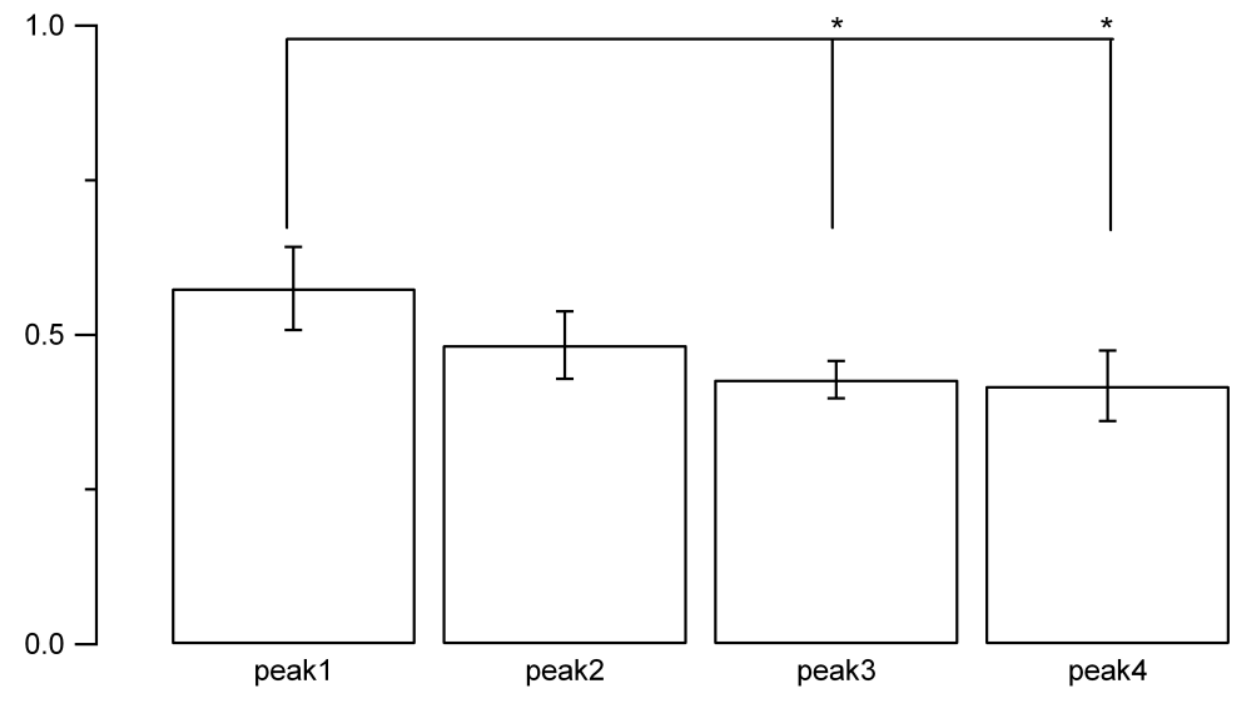

Figure 13 The Kyn blocking efficiency of EPSCs under $10 \mathrm{mM}$ TEA

The remaining fraction of EPSC after application of Kyn $(0.5 \mathrm{mM})$ is shown. The EPSC amplitude of each peak with Kyn was divided by that of the control group. The figure showed the average ratio of the $1^{\text {st }}$ to the $4^{\text {th }}$ peak over all connections we recorded. The blocking efficiency of the $1^{\text {st }}$ to the $4^{\text {th }}$ peak was $0.57 \pm 0.06,0.48 \pm 0.05,0.42 \pm 0.03,0.42 \pm 0.06$, respectively. There is a significant difference between the $1^{\text {st }}$ peak and the $3^{\text {rd }}$, the $4^{\text {th }}$ peaks. (paired t-test, $1^{\text {st }}$ v.s. $2^{\text {nd }}: p=0.09 ; 1^{\text {st }}$ v.s. $3^{\text {rd }}: p=0.01 ; 1^{\text {st }}$ v.s. $4^{\text {th }}: p=0.01$ ). The effect of Kyn 
was weaker than that of S1 under control condition, indicating that MVR was not as prominent at V1 as S1 even through Pr was increased by TEA.

We also analyzed the blocking effect in an alternative way by plotting the depression curve of the train stimulation trace in Figure 14. Here the EPSC amplitude was normalized to the first EPSC amplitude of each stimulus train. Again, in S1 the depression curve was significantly deeper under Kyn, indicating that receptor saturation reduced synaptic depression.
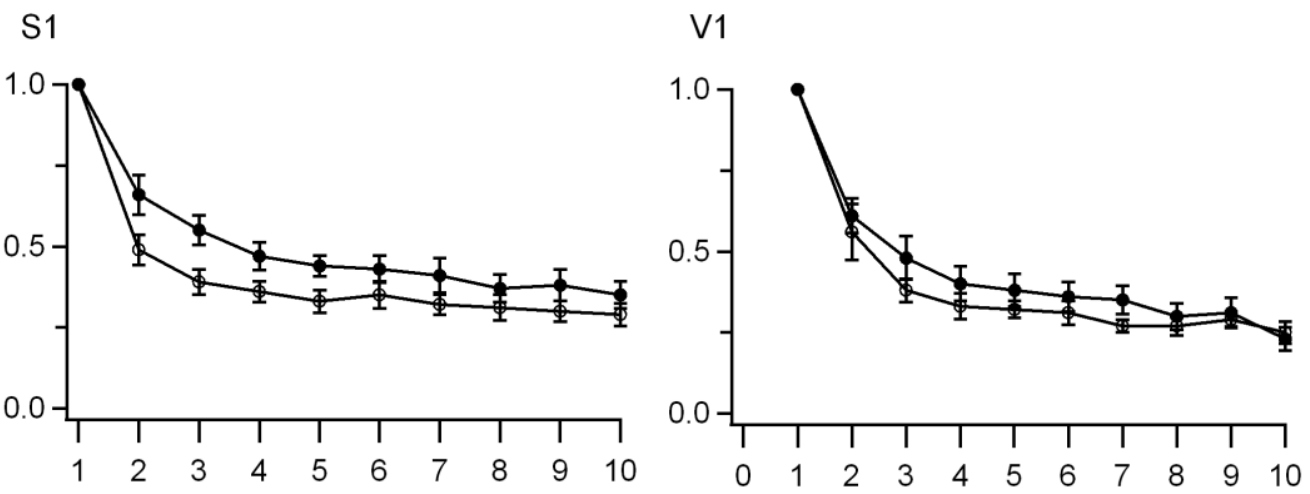

Figure 14 The effect of Kyn on the short-term plasticity of RS-RS connections in both cortices

The depression curve of synaptic transmission is shown. The EPSCs of each stimulus are normalized to the first one. The black solid circles represent the control group, and the black hollow circles represent the group under Kyn. The left panel shows the results from S1, and the right one shows the results from V1. In S1, there was significant difference (paired t-test, $\mathrm{p}<$ 0.05) between control group and the condition under Kyn whereas in V1 there was no significant difference (paired t-test, $\mathrm{p}>0.1$ ) between the two groups.

Consistently, when we calculated paired pulse ratio (PPR) of the first two EPSCs in a train, Kyn reduced the PPR at S1 but not V1 (Fig. 15). 


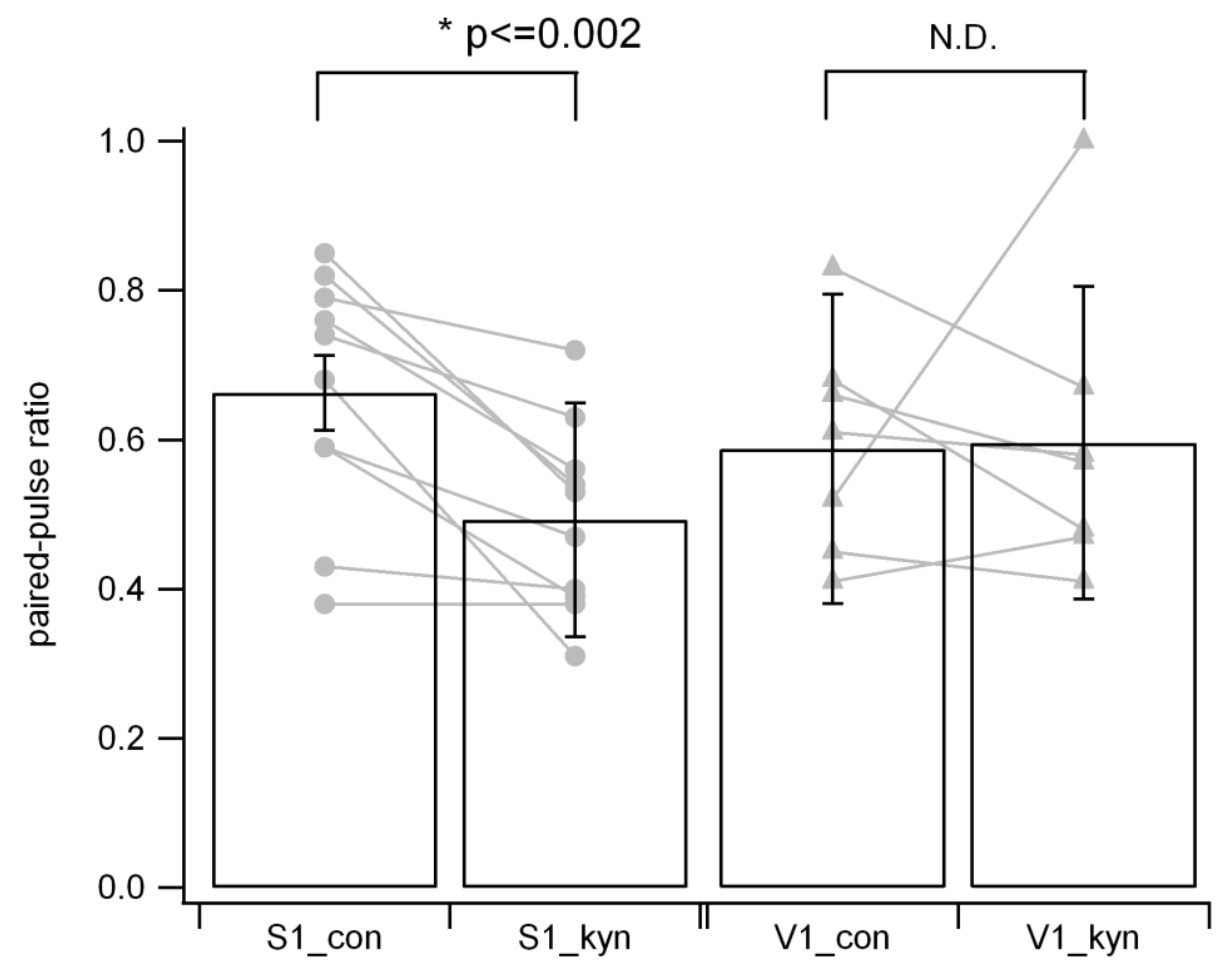

Figure 15 PPR with and without kyn

In each cell, PPR of the EPSCs was calculated using the first 2 EPSCs during a $50 \mathrm{~Hz}$ train and was compared with and without Kyn. At S1 (left) PPR decreased significantly (paired t-test, $\mathrm{p}<=0.02$ ) under Kyn, whereas there was no systematic tendency at V1. The result is consistent with the idea that MVR takes place at S1.

These results together with Fig. 9 suggested that postsynaptic receptor saturation reduced the synaptic depression significantly, making the synapse more stable during the train. However, V1 connections did not exhibit postsynaptic receptor saturation under the control condition.

\subsection{S1 connections can induce a postsynaptic AP more reliably than V1 connections.}

The results above suggested that RS-RS connections in S1 are more reliable than those in V1 because of MVR, higher Pr and postsynaptic receptor saturation. It remains to be tested how reliably a presynaptic AP can induce a postsynaptic AP. To test this 
issue, we first conducted simultaneous double patch recordings and examined if a presynaptic AP could induce a postsynaptic spike under current clamp mode. However, while in both S1 and V1 only EPSPs were observed, no postsynaptic spikes were elicited upon a single AP (data not shown), in contrast to previous studies (in S1 at young rats, see Egger et al., 1999; Feldmeyer et al., 1999). This indicates that the input from a single neuron is not sufficient to trigger APs at a postsynaptic neuron; more inputs might be required for postsynaptic firing. Due to the technical difficulty of conventional electrophysiology, only limited number of cells can be patched at the same time. Therefore, instead of multiple recordings, we performed dynamic clamp (Prinz et al., 2004) to systematically change the number of "presynaptic neurons". Dynamic clamp is different from voltage clamp and current clamp because instead of "clamping" the membrane potential or current, conductance is applied to the cell, and the amount of current flow depends on the instantaneous membrane potential. In our case this method has the advantage that a postsynaptic neuron can be excited by "virtual" presynaptic inputs, which therefore precludes the need to patch several presynaptic neurons at the same time and hence test the reliability of producing spikes at the postsynaptic neuron. To mimic the real conductance change at a postsynaptic neuron upon an AP at a presynaptic neuron, the average EPSCs measured at the soma of the postsynaptic neuron in the previous sets of experiments were used for the waveform of a unitary synaptic conductance and in calculating the mean peak amplitude and its variance. The first EPSCs during train stimulations were taken, and were fitted with a two-exponential function.

$$
\begin{aligned}
& \mathrm{g}=\mathrm{G} *\left[-\exp \left(-\mathrm{t} / \tau_{\mathrm{ris}}\right)+\exp \left(-\mathrm{t} / \tau_{\mathrm{dec}}\right)\right] \\
& \tau_{\mathrm{ris}}: \text { rising time constant; } \tau_{\mathrm{dec}}: \text { decay time constant }
\end{aligned}
$$

The mean and standard deviation (S.D.) of peak conductance change was also calculated from these EPSCs. Because these EPSCs were measured from one connected pair, the peak amplitude and variance were defined as a unit conductance change. The values from $\mathrm{S} 1$ and V1 cortical areas are shown in Table 2. 


\begin{tabular}{ccc}
\hline & $\mathrm{V} 1$ & $\mathrm{~S} 1$ \\
\hline mean of $\tau_{\text {ris }}$ & $600 \mu \mathrm{s}$ & $600 \mu \mathrm{s}$ \\
\hline mean of $\tau_{\mathrm{dec}}$ & $2 \mathrm{~ms}$ & $3 \mathrm{~ms}$ \\
\hline mean of peak conductance & $530 \mathrm{pS}$ & $700 \mathrm{pS}$ \\
\hline S.D. of peak conductance & $110 \mathrm{pS}$ & $115 \mathrm{pS}$ \\
\hline
\end{tabular}

Table 2 The parameters used to produce a conductance change waveform in V1 and S1 connections

We assumed that multiple inputs were linear summation of the unitary synaptic conductance, and they all arrived at the soma at the same time. This assumption may be oversimplistic compared to the real situation where inputs are not necessarily synchronous. However, it has been shown that L4 neurons can be simultaneously activated under physiological conditions (Petersen and Sakmann, 2001). Different number of inputs (presynaptic neurons) (from 4 cells to 40 with an increased step of 4) was applied simultaneously at a postsynaptic neuron (resting membrane potential was held at approximately $-50 \mathrm{mV}$ ). For each number of inputs, 50 stimuli were given at an interval of $200 \mathrm{~ms}$, and the EPSPs elicited upon the conductance change were recorded at a postsynaptic cell. Figure 16 shows an example trace recorded from a post-synaptic neuron. 


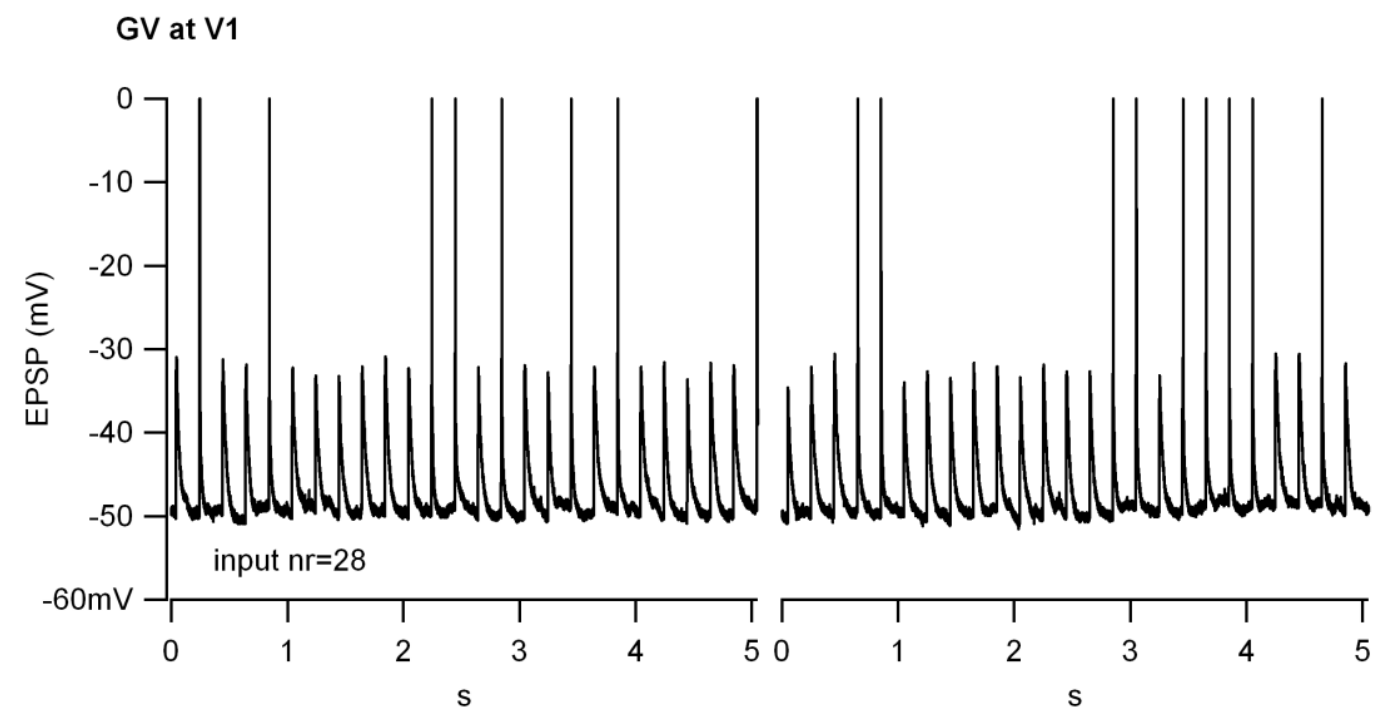

Figure 16 An example trace of EPSPs recorded from a post-synaptic V1 RS neuron

The conductance of V1 (GV) was injected to a RS neuron in V1 to induce EPSPs. In this example, 28 inputs were given, and the post-synaptic neuron exhibited a mixture of graded EPSPs and APs. The difference between an EPSP and an AP is significant, so one can count the number of APs without confusion. In total 50 stimuli were given with a $200 \mathrm{~ms}$ interval. However, due to the software limitation, only 25 stimuli can be recorded at one time and the protocol was repeated twice to obtain 50 peaks. The first 25 -stimuli indeed contain 26 peaks, but the last peak was not counted because it was not fully recorded. In this specific case, there are 16 APs out of 50 stimuli; therefore the AP probability is $32 \%$.

First we compared the threshold number of inputs required to elicit APs at the postsynaptic neuron in both cortices (Fig.17A). Here, threshold was defined as the minimum number of inputs required to induce an AP spike during the entire protocol (50 stimuli). In V1, some postsynaptic neurons did not fire any AP till the highest number of synaptic inputs we applied to the cell, so in these cases, the highest number plus 4 were given for the threshold number. As figure 17A shows, the threshold number of S1 connections (13 inputs) was significantly lower than that of V1 connections (28 inputs). Next, the probability of AP firing upon stimulation was plotted against the number of inputs in Fig. 17B. The AP probability is calculated from the number of APs elicited during 50 stimuli (See also Fig.16). Clearly, more APs were observed upon the same number of inputs at $\mathrm{S} 1$ connections than at V1 ones. Therefore, either (1) the synaptic conductance at $\mathrm{S} 1$ induces more firing or (2) the intrinsic membrane properties are 
different and the S1 neurons fire more easily. To study the effect of the former, synaptic conductance of S1 and V1 were injected into the same neuron (either S1 or V1) in the next set of experiments. Figure $17 \mathrm{C}$ and D show that the S1 conductance induced APs more reliably at both S1 and V1 postsynaptic neurons. This result can be further quantified by fitting the data of Fig. 17 with a Hill function, and $n$ (steepness of the inputoutput function) and half maximum (the number of neurons needed to excite the AP with probability of $50 \%$ ) were estimated (see Table 3). GS and GV are the synaptic conductance of S1 and V1, injected to either S1 or V1 neuron, respectively. GS to S1 needs 14 neurons and input-output has a steep function whereas GV to V1 is much less effective and has a shallow function. Because GV to S1 needs twice as many neurons as GS to S1 and has a shallower input-output relationship, difference in the synaptic conductance does have an important consequence on the firing. At the same time, GS to V1 needs $50 \%$ more neurons to reach half maximum (24 neurons) and the input -output relationship becomes shallower compared with the condition when GS was injected to S1 neuron, difference in intrinsic membrane properties does contribute to the AP firing. These results strongly support the argument that $\mathrm{S} 1$ connections are more reliable than V1 connections due to their synaptic properties. 

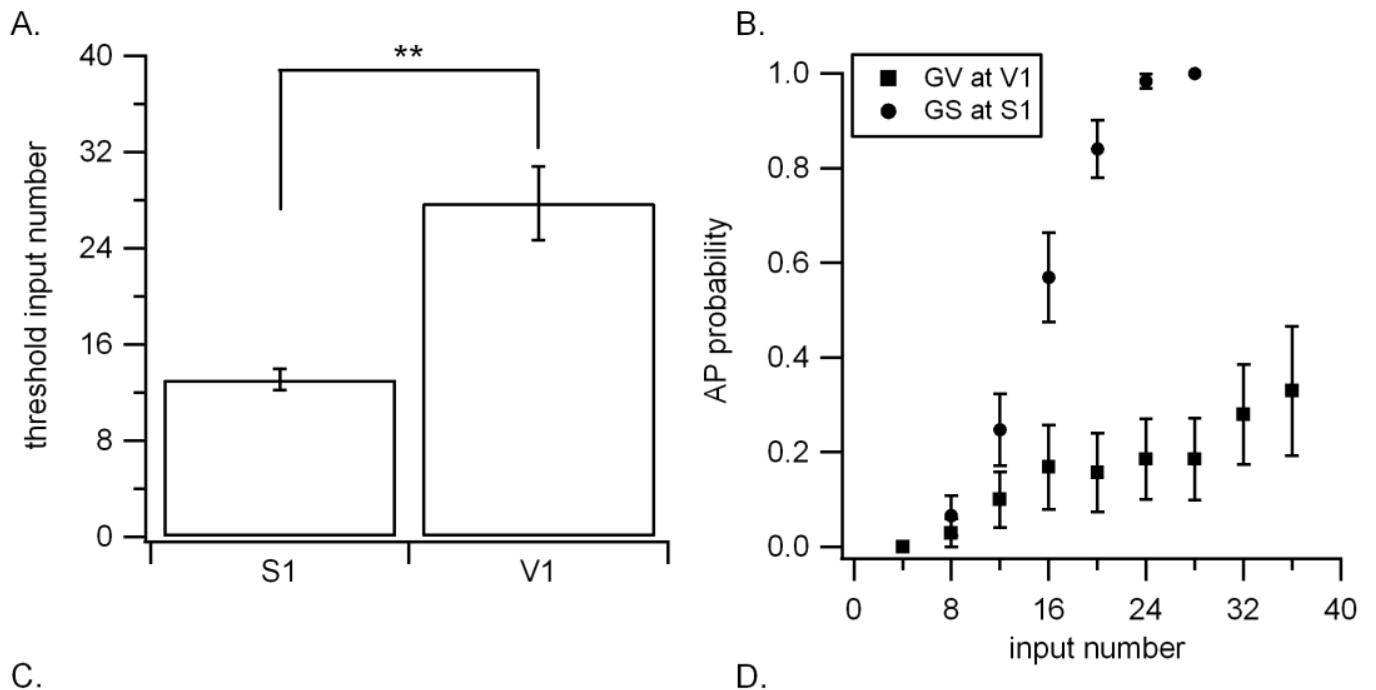

C.

D.
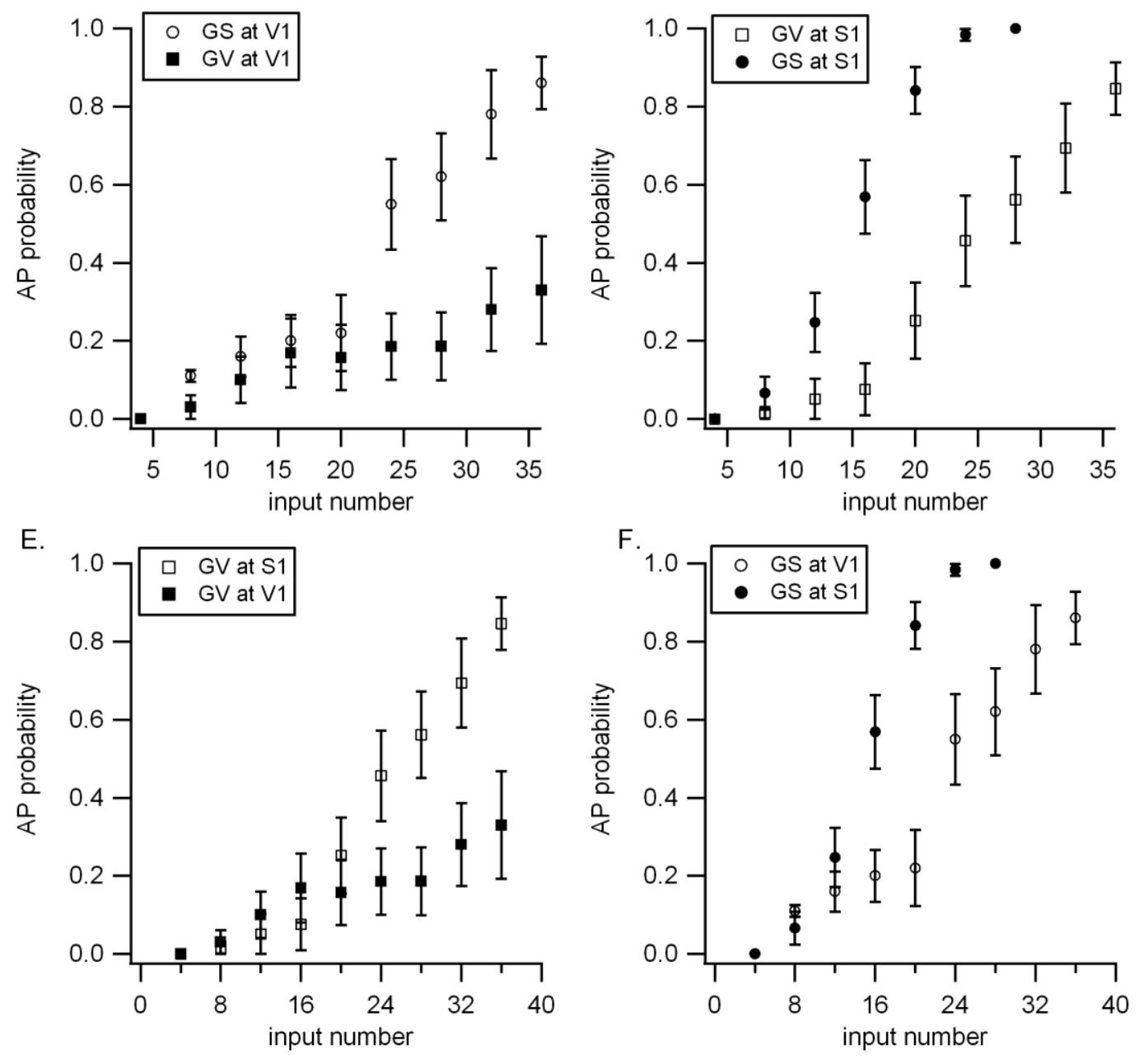


\section{Figure 17 S1 connections induce postsynaptic spikes more than V1 connections}

Dynamic clamp was used to examine the consequence of the different synaptic features between the two cortical areas on the postsynaptic AP firing. GS and GV are the synaptic conductance obtained from S1 and V1, respectively.

(A) The threshold input number required to elicit APs at a postsynaptic neuron in S1 and V1, when GS and GV were applied to S1 and V1, respectively. The threshold in S1 was significantly lower than that in V1 (t-test, $\mathrm{p}<=0.0002$ ). (B-F) The probabilities of firing APs at a postsynaptic neuron upon different input number were plotted. The solid circles represent the AP probability when S1 postsynaptic neurons were stimulated with S1 input parameters (with conductance change measured from $S 1$ connections) ( $n=16$ to 22 cells; $n=5$ cells for the data point of 28 inputs). The hollow circles represent the AP probability when V1 postsynaptic neurons were stimulated with $\mathrm{S} 1$ input parameters ( $\mathrm{n}=3$ to 9 cells). The solid squares represent the AP probability when V1 postsynaptic neurons were stimulated with V1 input parameters ( $n=8$ to 16 cells, $n=3$ cells for the data point of 40 inputs). The hollow squares represent the AP probability when S1 postsynaptic neurons were stimulated with V1 input parameters ( $\mathrm{n}=7$ to 15 cells).

\begin{tabular}{ccc}
\hline & $\mathrm{N}$ & half $\max$ \\
\hline GS to $\mathrm{S} 1$ & 5.6 & 14.9 \\
\hline GV to V1 & 1.4 & 64.0 \\
\hline GS to V1 & 3.8 & 23.9 \\
\hline GV to S1 & 4.4 & 25.8 \\
\hline
\end{tabular}

Table 3 The parameters estimated by fitting the data of Fig. 13 with a Hill function

There are three differences in the conductance waveform parameters between S1 and V1: the peak amplitude, decay time constant, and fluctuation (S.D.). We isolated the effects of decay time constant and fluctuation in Fig. 18 by re-plotting the AP probability against the amplitude of synaptic conductance, instead of the input number. While the AP probability was not significantly different between applying GV and GS at the V1 postsynaptic neurons (data not shown), it showed a difference at the S1 postsynaptic 
neurons (Fig. 18A). In this plot, GS induces more firing in S1 neurons. When the data were quantified by a Hill function, the GS data could be fitted with $n=5.6$ and half maximal of $10410 \mathrm{pS}$ whereas the GV data could be fitted with $\mathrm{n}=4.4$ and half maximal of $13674 \mathrm{pS}$, indicating that the difference of response in S1 neurons is approximately 30 $\%$. Furthermore, we found that decreasing the decay time constant of GS from $3 \mathrm{~ms}$ to 2 ms decreased the AP probability in S1 (Fig. 18B). When the data were fitted by a Hill function, the $3 \mathrm{~ms}$ data could be fitted with $\mathrm{n}=5.6$ and half maximal of $10411 \mathrm{pS}$, whereas the $2 \mathrm{~ms}$ data could be fitted with $\mathrm{n}=5.5$ and half max of $13769 \mathrm{pS}$, indicating the rightward shift of the relationship by $30 \%$, which showed that the decay time constant of synaptic conductance was responsible for most of the difference in figure 18A. Given that GV and GS had a difference in the half maximal input number by $70 \%$ (Fig 17D), approximately $30 \%$ of the difference of the total difference shown in Fig 17D could be explained by different synaptic decay time constant and the rest (40\%) was from the unit conductance amplitude of one input. On the other hand, fluctuation of the amplitudes, which is determined by the transmitter release fluctuation and the postsynaptic receptor saturation, had only a minor effect (Fig. 18C, D). In this experiment, in addition to the original GS, the synaptic conductance assuming no receptor saturation was used. The parameter was calculated by using SD obtained under the kyn condition, but scaling the mean and SD up by the kyn blocking efficiency (mean of $760 \mathrm{pS}$, SD of $200 \mathrm{pS}$ ). In conclusion, the peak synaptic conductance (determined by MVR and Pr) as well as the synaptic decay (determined postsynaptically) make a difference in the postsynaptic AP firing between S1 and V1. 
A.

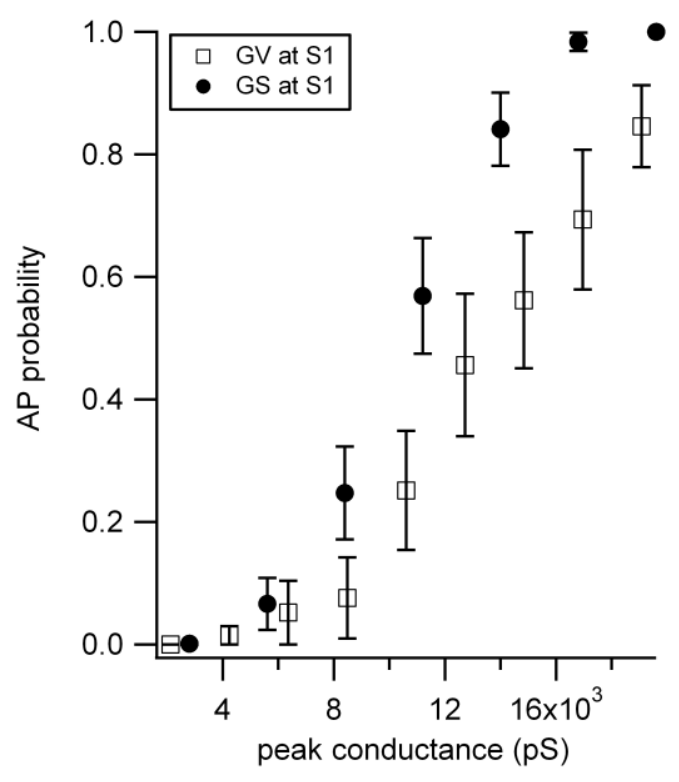

C.

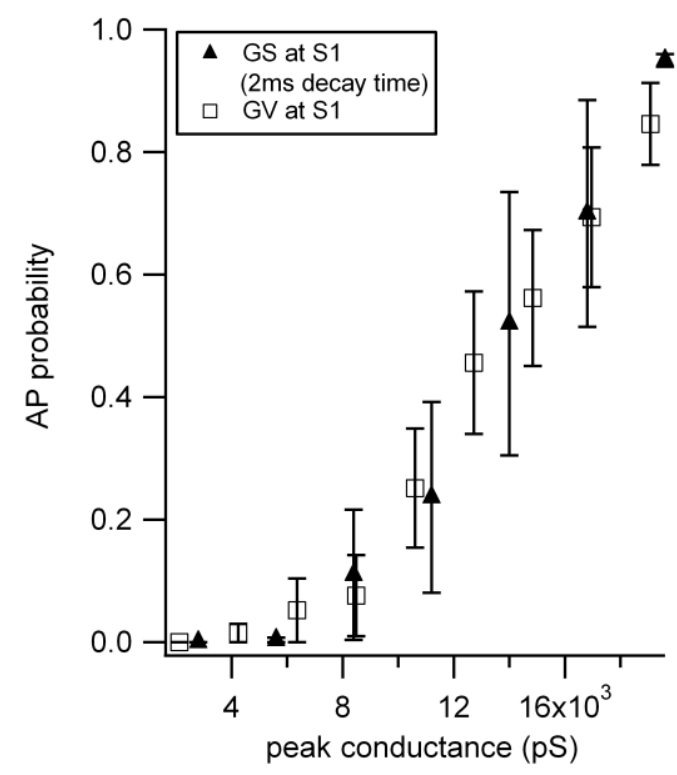

B.

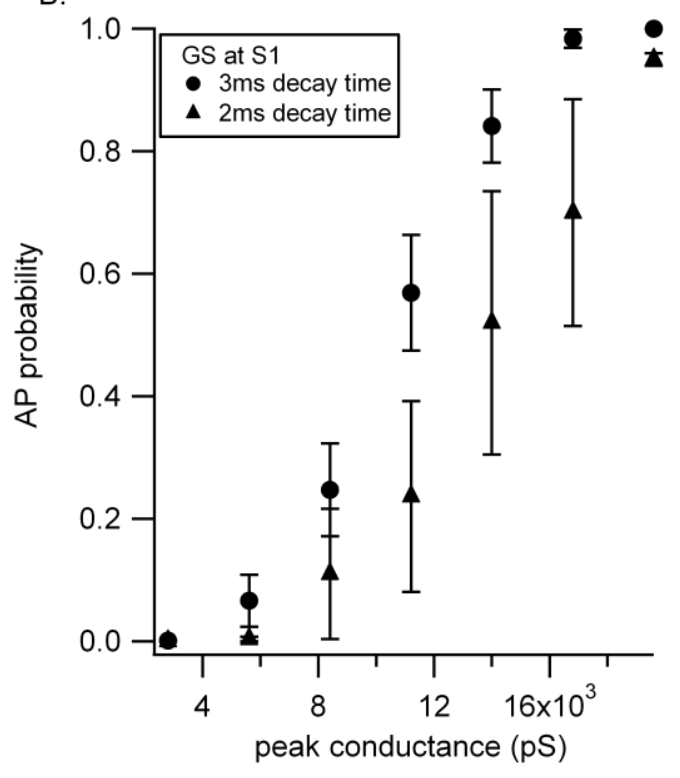

D.

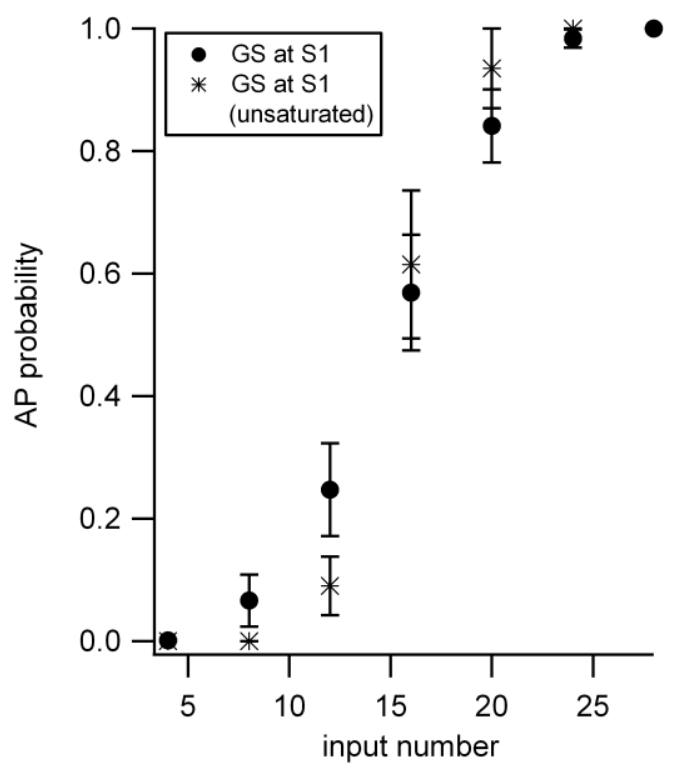

Figure 18 The effects of decay time constant and fluctuation of the postsynaptic responses on the postsynaptic AP firing at the S1 neuron.

The same experiment as Fig 17, using dynamic clamp. (A) The data are the same as Fig 17D, but probability of AP firing at the S1 neuron is plotted against the peak amplitude of synaptic conductance, thereby canceling the effect of a difference in the amplitude between GV (synaptic conductance from V1) and GS (synaptic conductance from S1). The same plot at the V1 neuron showed no major difference between GV and GS (data not shown). (B) The 
synaptic decay time constant of $2 \mathrm{~ms}$ or $3 \mathrm{~ms}$ was used. Other parameters remained the same as the original GS. The number of synaptic inputs was varied and was applied to the S1 neuron. The $3 \mathrm{~ms}$ decay could induce more spikes, indicating that the decay time constant had a consequence on the postsynaptic firing. (C) GS with the decay time constant of $2 \mathrm{~ms}$ (the same data as B) and GV (originally the decay time constant of $2 \mathrm{~ms}$ ) were applied to the $\mathrm{S} 1$ neuron. The AP probability is plotted against the peak conductance. The data overlap, suggesting that the $\mathrm{GV}$ is less reliable to induce the postsynaptic AP because of faster synaptic decay, in addition to the smaller peak amplitude. (D) In this panel, the effect of synaptic fluctuation was tested. Because of postsynaptic receptor saturation, GS fluctuates less than the condition without saturation. The two conditions had almost no difference (except a small difference at the low end of the input-output relationship), indicating relatively minor effect of the receptor saturation on the postsynaptic AP firing.

When Fig. 17B and C, D were compared, the differences seem to be more pronounced in Fig. 17B. In addition, Fig $17 \mathrm{E}$ and $\mathrm{F}$ show that the same conductance parameters (irrespective of $\mathrm{V} 1$ or $\mathrm{S} 1$ ) elicited higher AP more effectively at the S1 postsynaptic neuron compared with V1 neuron. GS to V1 needs $50 \%$ more input number (S1: 16 inputs v.s. V1: 24 inputs) to reach half maximum response; whereas GV to V1 requires even much more than 50\% input number to reach half maximum response (until 40 input number of GV, V1 neurons did not reach half maximum response, so the exact half maximal number of inputs was not calculated). Therefore, factors other than the synaptic conductance (such as the intrinsic membrane properties) do affect reliable AP firing at the S1 neuron upon presynaptic firing. We quantified the data of Fig. 17 with a Hill function (see above, Table 3) and found that contribution of synaptic conductance and intrinsic membrane properties contribute equally to more effective AP firing at S1. One possible source could be the input resistance of the neurons. We measured the input resistance, as shown in Fig. 19, and found no significant difference between S1 RS neurons and V1 RS neurons (Fig. 19C). Furthermore, the input threshold was not dependent on the input resistance in both cortical neurons (Fig. 19A, B). The data indicates that input resistance does not contribute much to the reliability of the AP firing at the postsynaptic cell. Therefore, the intrinsic membrane properties other than input resistance at the postsynaptic neuron are important to make $\mathrm{S} 1$ connections more reliable than V1 connections which, however, remain to be investigated in future detailed studies. 
A.

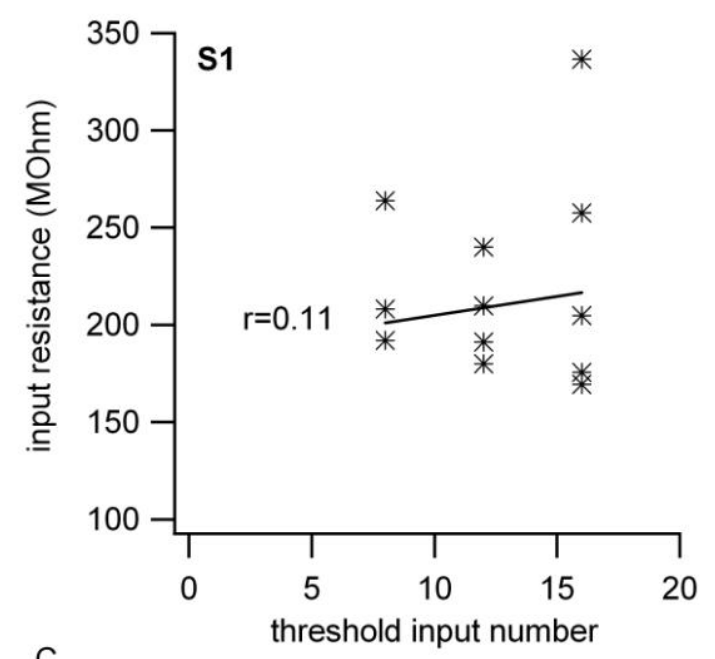

C.

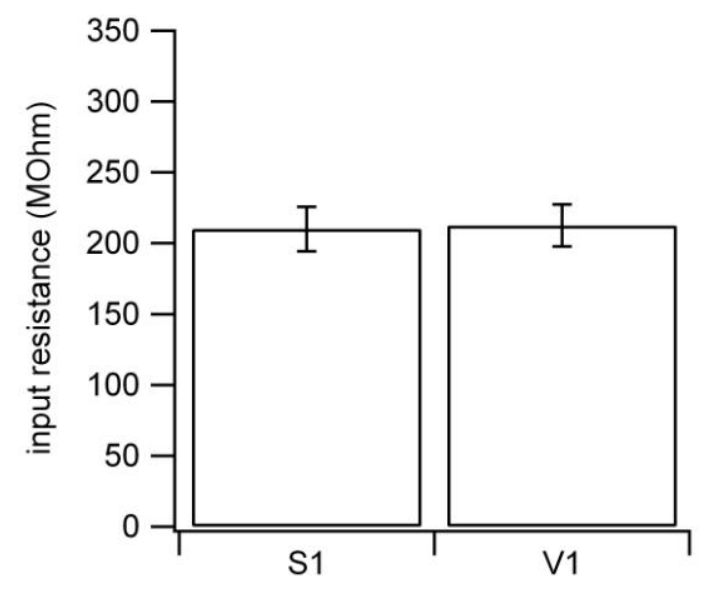

B.

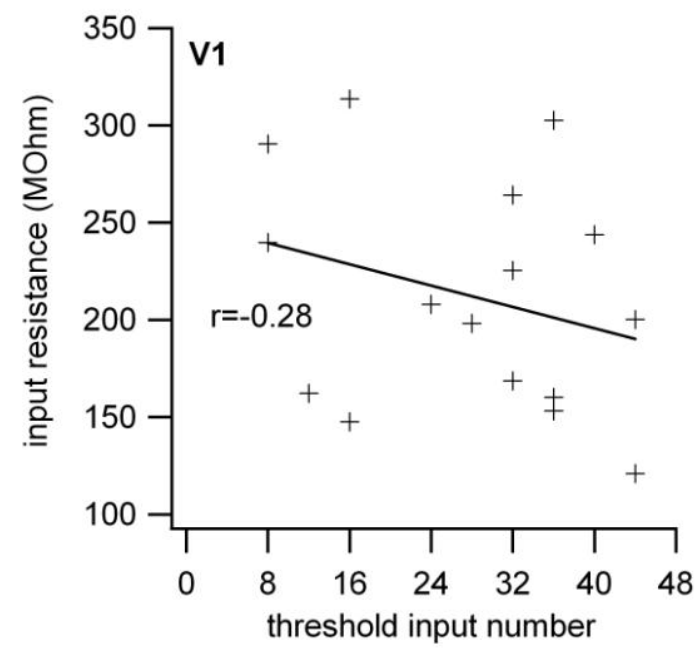

Figure 19 Input resistance of RS neurons in S1 and V1

(A) The correlation plot of input resistance of each RS neuron in S1 and the threshold number of inputs required to elicit APs. The correlation coefficient is 0.11. (B) The correlation plot of input resistance of each RS neuron in $\mathrm{V} 1$ and the threshold number of inputs required to elicit APs. The correlation coefficient is -0.28. (C) The input resistance measured from RS neurons in S1 and V1. The mean input resistance of RS neurons in S1 is 209.97 \pm 15.8 MOhm ( $n=13)$ whereas that of RS neurons in V1 is $212.49 \pm 14.81 \mathrm{MOhm}(\mathrm{n}=16)$. There is no significant difference between the input resistance of these two cortices ( $t$-test, $p<=0.91$ ). 


\section{Discussion}

We have identified quantal parameters in two primary sensory cortical areas using two independent methods, $\mathrm{V}-\mathrm{M}$ analysis and the pool depletion protocol. Pr in V1 neurons was quite heterogeneous in comparison to S1. Furthermore, we observed MVR in RS-RS connections in L4 in S1, whereas in V1 a single release site released a single vesicle under physiological condition. Together with postsynaptic receptor saturation characteristics, the behavior of a synapse in the two cortical regions can be well described as follows. In response to a presynaptic AP, the $\mathrm{S} 1$ connections are more reliable to induce the postsynaptic APs due to the high Pr, MVR, and possibly the slower synaptic decay and the intrinsic membrane properties. During a train of APs, the receptor saturation in addition to high vesicle replenishment rates compensates for vesicle pool depletion such that presynaptic APs can cause robust postsynaptic responses.

\subsection{Multivesicular release at cortical synapses}

The theory of release process at central synapses is classified into two: one is "onerelease site, one-vesicle" hypothesis, which means at most one vesicle would be released at one release site upon an AP (Korn et al., 1981; Silver et al., 2003), and the other is MVR which means more than one vesicle can be released at one release site (Tong and Jahr, 1994). In large synapses such as the calyx of Held (Taschenberger et al., 2002), climbing fiber in cerebellum (Wadiche and Jahr, 2001) and ribbon synapses (Glowatzki and Fuchs, 2002; Li et al., 2009; Singer et al., 2004), MVR was commonly observed. Multivesicular release was also found at more conventional synapses such as hippocampal Schaffer collateral-CA1 synapses (Oertner et al., 2002). However, at cortical synapses it is suggested that release is constrained to a single vesicle per AP 
(Biro et al., 2005; Buhl et al., 1997; Egger et al., 1999; Feldmeyer et al., 1999; Gulyas et al., 1993; Korn et al., 1981; Silver et al., 2003). One study reported MVR at L2/3 neurons in V1 at room temperature (Watanabe et al., 2005), which was in contrast with other reports in S1 (Koester and Johnston, 2005; Rozov et al., 2001). The other study recently suggested that MVR occurred at L5 synapses in S1 (Loebel et al., 2009). Here, our data provide strong evidence in favour of MVR at RS-RS connections of L4 in S1 under physiological conditions based on the differential block of Kyn, which clearly indicates that the local glutamate transient changes during a train of APs. Because synaptic contacts are spatially separated (Feldmeyer et al., 1999), glutamate spillover to the neighboring synapses is highly unlikely. Furthermore, significant postsynaptic receptor saturation was indicated from the variance-mean analysis, which is consistent with MVR. The single vesicle hypothesis was often postulated based on the demonstration that the functional $\mathrm{N}$ matched with the anatomical $\mathrm{N}$ (Biro et al., 2005; Buhl et al., 1997; Gulyas et al., 1993; Korn et al., 1981; Silver et al., 2003). While it is definitely informative to make such comparisons between anatomy and physiology, a strong correlation between the two numbers does not necessarily imply that only a single vesicle can be released at individual release site upon one AP. This only holds true by assuming that the release-ready vesicles are evenly distributed among every anatomical contact. At central synapses, this assumption is not always supported because $\mathrm{Pr}$ is rather heterogeneous among transmitter release sites (Murthy et al., 1997; Rosenmund et al., 1993). Without testing the glutamate concentration at the release site, one cannot conclude that only one vesicle is released at one site upon one AP (Silver et al., 2003). Moreover, the binomial model and variance-mean analysis both require the assumption that postsynaptic receptors are linear indicators of transmitter release. If there is receptor saturation, then the assumption would not be satisfied and one might underestimate $\mathrm{N}$ in such a scenario. The functional $\mathrm{N}$ of L4 connections in $\mathrm{S} 1$ (6 to 8) was twice as many as the anatomical synapse number in rats (3 to 4) (Feldmeyer et al., 1999), which is consistent with MVR. Therefore, assuming anatomical $\mathrm{N}$ as functional $\mathrm{N}$ in RS-RS connections in L4 of S1 (Egger et al., 1999) might be misleading. At hippocampal and cerebellar synapses, a synaptic bouton contains a number of docked vesicles (Schikorski and Stevens, 1997; Xu-Friedman et al., 2001). At cortical synapses, number of docked 
vesicles per active zone has not been measured precisely. A direct correlation between the number of docked vesicles and the number of releasable vesicles per synapse remains to be resolved.

On the other hand, RS-RS connections of L4 in V1 did not show differential block by Kyn, indicating a single vesicle release under physiological condition. Since the Pr in $\mathrm{V} 1$ is lower than that in $\mathrm{S} 1$ on average, we tested whether those connections could exhibit MVR when the Pr was increased. Indeed, differential block by Kyn was observed when TEA was applied, suggesting that a single release site could support release of more than one vesicle per site upon a single AP at high Pr in V1. This argues against the existence of a single vesicle release constraint at least in RS-RS connections. It is important to note that even under high Pr, the differential block was less pronounced compared with S1. This means that apart from the $\mathrm{Pr}$, the synapses at the somatosensory cortex intrinsically favour MVR. Nevertheless, the difference in $\operatorname{Pr}$ and degree of postsynaptic receptor saturation distinguishes the same type of connections in two cortical areas from each other. Because of the receptor saturation, S1 connections may be less prone to synaptic plasticity such as long-term potentiation (Egger et al., 1999) whereas the V1 connections are more modifiable upon different stimuli.

\subsection{Differences of synapses at two sensory areas and their physiological implication}

\subsubsection{Differences in Pr and replenish rate}

The connections in $\mathrm{V} 1$ show heterogeneous $\mathrm{Pr}$ whereas those in $\mathrm{S} 1$ are more homogeneous. There could be several reasons for this disparity. The $\mathrm{Ca}^{2+}$ sensor of the release machineries for the two systems could be different. It is possible that the distance

between the vesicles and $\mathrm{Ca}^{2+}$ channels in $\mathrm{V} 1$ connections is more variable than that in S1 connections. The cell morphology in the two cortical regions could be different. The location of synaptic contact in V1 might be more diverse than in S1, or the axon arbor could be wider in V1. In addition to Pr, the vesicle replenishment rates differ from V1 to 
S1 neurons. Upon a strong depolarization (depletion pulse), vesicle replenishment rates in $\mathrm{S} 1$ were $\sim 3$ times faster than those in V1 ( $\mathrm{T}_{\text {rec }}$ in $\mathrm{S} 1: 30 \mathrm{~ms} ; \mathrm{T}_{\text {rec }}$ in $\mathrm{V} 1: 100 \mathrm{~ms}$ ). In contrast, when we fit the depression curve of the train stimulation, the replenishment rates were the same in both cortical regions ( $\mathrm{T}_{\text {rec }}$ of both: 100ms). During a long depolarization, there is a massive presynaptic $\mathrm{Ca}^{2+}$ influx, which most likely speeds up the replenishment process in $\mathrm{S} 1$, whereas in $\mathrm{V} 1$ this process is saturated during a $50 \mathrm{~Hz}$ stimulation. Nevertheless, the vesicle replenishment rate is much faster than retinal bipolar cells (Mennerick and Matthews, 1996) and the calyx of Held (Wu and Borst, 1999) (the order of seconds), and is comparable to mossy fiber and parallel fiber synapses in cerebellum (Crowley et al., 2007; Saviane and Silver, 2006) as well as hair cell synapses (Griesinger et al., 2005; Li et al., 2009; Moser and Beutner, 2000). Since a prolonged depolarization did not release more vesicles compared to an AP train, no reluctant vesicles exist at the L4 synapse (Hallermann et al., 2003; Mennerick and Matthews, 1996; Neher and Sakaba, 2001). Thus, the cortical synapses with a limited number of functional release sites may be able to maintain effective transmission by fast vesicle replenishment together with a high release probability.

\subsubsection{Difference in reliability of synaptic transmission revealed by dynamic clamp}

The dynamic clamp experiment (Fig. 17, 18) further demonstrates that S1 synaptic conductance can elicit APs more reliably at the postsynaptic neuron. Therefore, the differences in synaptic parameters (high Pr and slower synaptic decay) enable more reliable AP firing at the postsynaptic neuron. To elicit an AP at the postsynaptic site, both S1 and V1 needed convergence of multiple presynaptic inputs (> 4, Fig. 17). This is consistent with the finding that multiple RS neurons can be activated simultaneously within the cortical column under more physiological condition (Petersen and Sakmann, 2000). However, Egger V. et al. have reported that one AP at the pre-synaptic neuron can fire AP at the post-synaptic one in the same type of connections in S1 (Egger V. et al, 1999). This discrepancy might due to the age of the animal. We used mice older than P18 whereas they used young rats (P14). The expression of ion channels can be altered 
during development, and this might result in different firing properties or different time courses of postsynaptic potentials elicited by released synaptic vesicles.

In order to isolate various factors that may contribute to the differential reliability in synaptic transmission between the two cortices, we re-plotted the AP probability against the absolute conductance amplitude instead of the input number. With this plot the difference in the "unit input conductance" between GV and GS was removed, and it is possible to look at the responses of different post-synaptic neurons as a function of the total conductance waveform (including the amplitudes, decay time constanst, and fluctuations of the peak amplitude of GS and GV). Here we define "unit input conductance" as the conductance contributed by one input (one pre-synaptic neuron). Fitting the plot with a Hill function, different responses to either GS or GV waveform were quantified. When either GS or GV was applied to the RS neurons of S1, GV required 70\% more input number than GS did to reach a half maximum response. This is the case when the AP probability was plotted against the number of input. However, only $30 \%$ more conductance was needed for GV after re-plotting the AP probability against the total conductance. The result suggests that the difference in the unit input conductance amplitude (Table 2, GV $530 \mathrm{pS}$ vs GS $700 \mathrm{pS}$ ) contributes approximately $40 \%$ of the differences when the S1 post-synaptic neurons were used for dynamic clamp. However, when either GS or GV was fed in V1 neurons instead, the situation was different: The input-output response curve at $\mathrm{S} 1$ neurons had a very steep function of either the number of inputs or the total conductance ( $\mathrm{n}$ in Hill function fitting of more than 4). In contrast at V1 neurons the slope was shallower and n (slope) of GS and GV differed more than two-fold (see Table $3, \mathrm{n}=3.8$ v.s. 1.4 when plotting AP probability against the input number). The difference seems to indicate that V1 neurons have distinct membrane properties from S1 neurons (see below). It is important to note that the responses of V1 neurons did not reach a half maximum even though 40 inputs (GV) were simultaneous applied. Because the input-output relationship does not reach a half maximum, quantitative comparison of the differences between GS and GV by fitting with a Hill function is not necessarily accurate for the V1 data. Nevertheless, after replotting the AP probability against the absolute conductance amplitude, there was no 
significant difference between the response curve of GS and GV (data not shown) whereas the difference existed when the AP probability was plotted against the input number. This indicates that the difference between GV and GS at V1 neurons mainly comes from the unit input conductance of the synaptic input. It remains possible that the input-output relationship (when plotting AP probability against the total conductance) of GV and GS matched with each other only at the low-end of the whole response curve, and the mechanisms other than the difference in the unit input conductance may contribute when $>40$ inputs are simultaneously activated. More experiments are needed to evaluate the effect of various factors on the AP responses at V1 neuron. Further analysis was only carried out in S1 neurons in this study (Fig. 18). Except for the peak conductance of one input, GV and GS waveforms also differ in the decay time constant and SD (fluctuation). Because altering the decay time of the simulated synaptic conductance by $1 \mathrm{~ms}$ made a 30\% difference on spike generation of S1 neurons (Fig 18B), and the SD of the synaptic conductance showed no significant effect on AP probability (Fig 18C), the decay time constant is mainly responsible for the $30 \%$ shift in figure 18A. This result suggested that the efficacy with which multiple synaptic inputs can elicit postsynaptic spikes at S1 neurons would be, in part, determined by the degree of synchrony between those inputs.

Other than synaptic mechanism for reliable signal transduction via a synapse, the intrinsic membrane properties are equally important. It is rather clear by looking at the slope (n) of the input-output relationship. The slope represents the gain of the inputoutput relationship at a post-synaptic neuron, and hence reflects the membrane firing property. Our result showed that $\mathrm{S} 1$ neurons exhibited a high gain $(\mathrm{n}=5)$ within our input range, and the slope of S1 neurons was always steeper than that of V1 neurons. On the other hand, the gain of V1 was shallower (Table 3). Therefore, the intrinsic membrane properties must be quite different between V1 and S1. S1 neurons can response reliably to inputs when more than 20 neurons are simultaneously activated, and make a large contrast in the input range between 10 and 20 neuron, amplifying the difference of signals around this range, In contrast V1 neurons seemed to respond more linearly to the number of input neurons, translating the incoming signals to the AP 
linearly. The input resistance of neurons in two cortices was similar, so we could exclude the impact of passive membrane properties near resting potential at least around soma. That V1 neurons have smaller gain might be resulted from less expression of $\mathrm{Na}^{+}$ channels or more expression of $\mathrm{K}^{+}$channels. It is also possible that in $\mathrm{S} 1$ neurons, the $\mathrm{Na}^{+}$channels are expressed with a higher density, and thus can boost up the membrane potentials to exceed the threshold for AP generation more easily. The gating and inactivation of ion channels (especially $\mathrm{Na}^{+}$and $\mathrm{K}^{+}$channels) expressed in neurons of these two cortices can be different as well. Moreover, only the currents ofAMPARs were used to simulate conductance change, but the summation of NMDARs currents was not considered in this study. It has been reported that about $40 \%$ of total EPSP in S1 L4 RS neurons is contributed by NMDAR (Feldmeyer et al., 1999). Considering that $1 \mathrm{~ms}$ difference in decay time constant of synaptic currents can already make a $30 \%$ shift in the input-output curve, the NMDAR can prolong the decay time even more and hence may change the response curve more extensively. On the other hand, Stern et al. showed that the ratio of NMDAR mediated EPSC is rather heterogeneous in different cells of V1 RS neurons, ranging from $15 \%$ to $40 \%$ (Stern et al., 1992) which is lower than the ratio in S1 RS neurons (Feldmeyer et al., 1999). Therefore, if we take the NMDAR conductance into account, the difference in gain between V1 and S1 RS neurons can be even more drastic., We should note a one caveat inherent to dynamic clamp: Since the conductance was injected at the soma of a neuron in the dynamic clamp mode, the active propagating role of the dendrites was neglected. Active conductance of the dendrites may have some effect on the input-output relationship; nevertheless, it does not alter our conclusion that V1 and S1 neurons have different post-synaptic membrane properties.

\subsubsection{Summary of physiological and anatomical data}

Furthermore, if we consider the morphological differences, the RS-RS network of L4 in S1 can integrate signals from various inputs into steady but specific outputs. This is because the neurons form a barrel structure (Feldmeyer et al., 1999; Petersen and Sakmann, 2000), and the intra-column connection rates are much higher than the intercolumn ones (20\%) (Lefort et al., 2009). On the other hand, the same network in V1 
does not show such strong integration in L4. Connectivity is very low (5\%, unpublished observation from our study, see also Rochefort et al., 2009) which makes coupling between neurons highly unreliable. Combining both morphological and physiological evidence together, we propose different models for signal transduction in L4 of S1 and V1 (Fig. 20). In S1, the sensory signals coming from thalamus first converge onto RS neurons to fire an AP. These RS neurons not only send out signals to L2/3 but also to other RS neurons in L4. This intra-layer connection may integrate with further signals coming from the thalamus, and thus can actively modulate the signal transduction within L4. Opposite to S1, RS neurons in V1 do not seem to have much interaction within L4. The rate of connectivity between RS neurons is low, and even if a pair of RS neurons is connected, the synaptic transmission across the synapse is unreliable. The Pr is heterogeneous, and the post-synaptic neurons require more presynaptic neurons to fire APs. Furthermore, we found that the RS-interneuron connectivity is much higher than that between RS neurons (preliminary findings), so one can expect that the feedback inhibition onto RS neurons within L4 can be dominating, which limits the AP firing only at the onset of sensory stimulation. The signals from the thalamus may just go through L4 of V1 without much modulation, and the response in L4 RS neurons is likely to be terminated only at the onset of stimulation. In a sense, the connections between excitatory neurons in L4 of mouse visual cortex may not be as important as those in S1. 
s1

Thalamocortical inputs

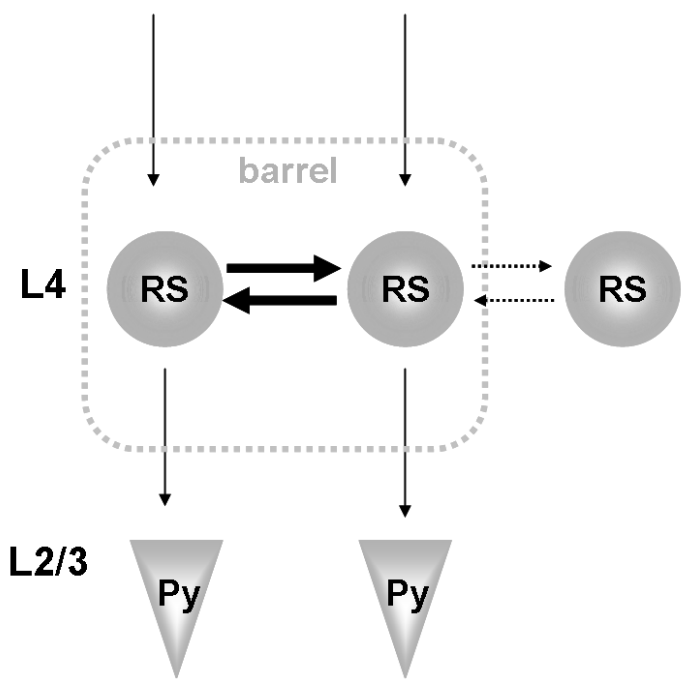

V1

Thalamocortical inputs

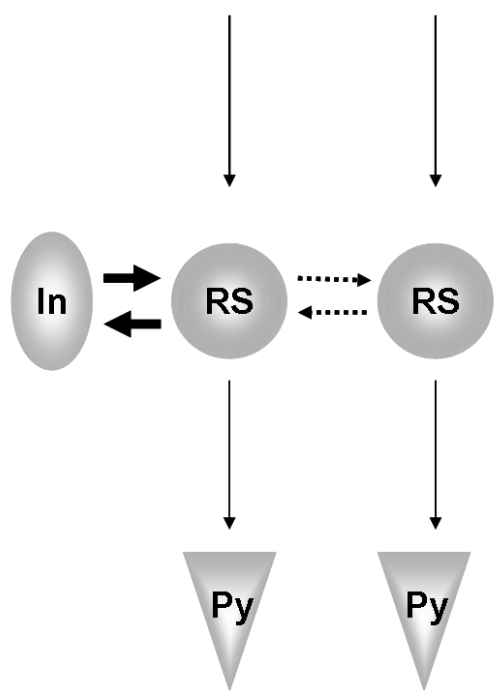

Figure 20 An illustration of different signal transduction models in L4 RS-RS connections between $\mathrm{S} 1$ and V1

The left panel shows a model for S1 RS-RS connections based on our results. Within a barrel the interaction between two RS neurons is reliable and strong (thick arrow) but the inter-barrel connections are weaker (thin and dotted arrow). The right panel shows a model for V1 RS-RS connections. Different from the S1 ones, RS-RS connections are rather weak in V1 (thin and dotted arrow). Instead, RS neurons have a stronger interaction with interneurons (In, thick arrow). In this study, because we did not investigate the thalamocortical input to L4 and the L4 output to pyramidal cells (Py) in L2/3, we illustrated as they are the same in S1 and V1. In real case, it may be different as well.

The anatomical structure of mouse visual cortex may not be representative for all spices, and vision for mice, mainly living in darkness, may not be not as crucial as that for cats or higher mammals. The role of L4 RS neurons in cat's or monkey's visual cortex may not be the same as that of those neurons in mouse visual cortex. Nevertheless, this study demonstrates that these differences in structural and physiological properties might correlate to the underlying functional tasks of the respective cortical areas. Moreover, using mice as an animal model has advantages of allowing genetic modification and more information at the molecular, and cellular level over higher mammals. Our study also provides a reference for bridging researches among different species. 


\section{Summary}

Neurons in layer 4 (L4) of the cortex play an important role in transferring signals from thalamus to other layers of the cortex. Understanding the fundamental properties of synaptic transmission between L4 neurons helps us to gain a clear picture of how the neuronal network in L4 co-operates to process sensory information. In the present study, we have determined the underlying parameters that govern synaptic strength such as quantal size (q), size of readily releasable vesicle pool (N) and release probability (Pr) of excitatory synaptic connections within L4 of the visual cortex (V1) and the somatosensory cortex (S1) in mice. While only a single vesicle is released per release site under physiological conditions at V1 synapses, multivesicular release (MVR) is observed at S1 synapses. In addition, we observed a saturation of postsynaptic receptors at S1 synapses. Other synaptic properties are similar in both cortices. Dynamic clamp experiments suggest that higher $\operatorname{Pr}$ and MVR at S1 synapses lower the requirement of the number of synaptic inputs to generate postsynaptic action potentials. In addition, the slower decay of synaptic current and the intrinsic membrane properties of the postsynaptic neuron also contribute to the reliable transmission between $\mathrm{S} 1$ neurons. 


\section{Bibliography}

Abbott, L.F., and Regehr, W.G. (2004). Synaptic computation. Nature 431, 796-803.

Abbott, L.F., Varela, J.A., Sen, K., and Nelson, S.B. (1997). Synaptic depression and cortical gain control. Science 275, 220-224.

Agmon, A., and Connors, B.W. (1991). Thalamocortical responses of mouse somatosensory (barrel) cortex in vitro. Neuroscience 41, 365-379.

Ahmed, B., Anderson, J.C., Douglas, R.J., Martin, K.A., and Nelson, J.C. (1994). Polyneuronal innervation of spiny stellate neurons in cat visual cortex. J Comp Neurol $341,39-49$.

Armstrong-James, M., and Fox, K. (1987). Spatiotemporal convergence and divergence in the rat S1 "barrel" cortex. J Comp Neurol 263, 265-281.

Beierlein, M., Gibson, J.R., and Connors, B.W. (2000). A network of electrically coupled interneurons drives synchronized inhibition in neocortex. Nat Neurosci 3, 904-910.

Beierlein, M., Gibson, J.R., and Connors, B.W. (2003). Two dynamically distinct inhibitory networks in layer 4 of the neocortex. J Neurophysiol 90, 2987-3000.

Binzegger, T., Douglas, R.J., and Martin, K.A. (2004). A quantitative map of the circuit of cat primary visual cortex. J Neurosci $24,8441-8453$. 
Biro, A.A., Holderith, N.B., and Nusser, Z. (2005). Quantal size is independent of the release probability at hippocampal excitatory synapses. J Neurosci 25, 223-232.

Buhl, E.H., Tamas, G., Szilagyi, T., Stricker, C., Paulsen, O., and Somogyi, P. (1997). Effect, number and location of synapses made by single pyramidal cells onto aspiny interneurones of cat visual cortex. J Physiol 500 ( Pt 3), 689-713.

Connors, B.W., and Gutnick, M.J. (1990). Intrinsic firing patterns of diverse neocortical neurons. Trends Neurosci 13, 99-104.

Cook, D.L., Schwindt, P.C., Grande, L.A., and Spain, W.J. (2003). Synaptic depression in the localization of sound. Nature 421, 66-70.

Crowley, J.J., Carter, A.G., and Regehr, W.G. (2007). Fast vesicle replenishment and rapid recovery from desensitization at a single synaptic release site. J Neurosci 27 , 5448-5460.

Diamond, J.S., and Jahr, C.E. (1995). Asynchronous release of synaptic vesicles determines the time course of the AMPA receptor-mediated EPSC. Neuron 15, 10971107.

Diamond, J.S., and Jahr, C.E. (1997). Transporters buffer synaptically released glutamate on a submillisecond time scale. J Neurosci 17, 4672-4687.

Dittman, J.S., and Regehr, W.G. (1998). Calcium dependence and recovery kinetics of presynaptic depression at the climbing fiber to Purkinje cell synapse. J Neurosci 18 , 6147-6162.

Dobrunz, L.E., and Stevens, C.F. (1997). Heterogeneity of release probability, facilitation, and depletion at central synapses. Neuron 18, 995-1008. 
Egger, V., Feldmeyer, D., and Sakmann, B. (1999). Coincidence detection and changes of synaptic efficacy in spiny stellate neurons in rat barrel cortex. Nat Neurosci 2, 1098-1105.

Feldmeyer, D., Egger, V., Lubke, J., and Sakmann, B. (1999). Reliable synaptic connections between pairs of excitatory layer 4 neurones within a single 'barrel' of developing rat somatosensory cortex. J Physiol 521 Pt 1, 169-190.

Ferster, D., and Lindstrom, S. (1983). An intracellular analysis of geniculo-cortical connectivity in area 17 of the cat. J Physiol 342, 181-215.

Foster, K.A., Kreitzer, A.C., and Regehr, W.G. (2002). Interaction of postsynaptic receptor saturation with presynaptic mechanisms produces a reliable synapse. Neuron $36,1115-1126$.

Foster, K.A., and Regehr, W.G. (2004). Variance-mean analysis in the presence of a rapid antagonist indicates vesicle depletion underlies depression at the climbing fiber synapse. Neuron 43, 119-131.

Frerking, M., and Wilson, M. (1996). Effects of variance in mini amplitude on stimulusevoked release: a comparison of two models. Biophys J 70, 2078-2091.

Gilbert, C.D. (1983). Microcircuitry of the visual cortex. Annu Rev Neurosci 6, 217-247.

Gilbert, C.D., and Wiesel, T.N. (1979). Morphology and intracortical projections of functionally characterised neurones in the cat visual cortex. Nature 280, 120-125.

Glowatzki, E., and Fuchs, P.A. (2002). Transmitter release at the hair cell ribbon synapse. Nat Neurosci 5, 147-154. 
Goffinet, F., Maillard, F., Mihoubi, N., Kayem, G., Papiernik, E., Cabrol, D., and Paul, G. (2003). Bacterial vaginosis: prevalence and predictive value for premature delivery and neonatal infection in women with preterm labour and intact membranes. Eur $\mathrm{J}$ Obstet Gynecol Reprod Biol 108, 146-151.

Griesinger, C.B., Richards, C.D., and Ashmore, J.F. (2005). Fast vesicle replenishment allows indefatigable signalling at the first auditory synapse. Nature 435, 212-215.

Gulyas, A.I., Miles, R., Sik, A., Toth, K., Tamamaki, N., and Freund, T.F. (1993). Hippocampal pyramidal cells excite inhibitory neurons through a single release site. Nature 366, 683-687.

Gupta, A., Wang, Y., and Markram, H. (2000). Organizing principles for a diversity of GABAergic interneurons and synapses in the neocortex. Science 287, 273-278.

Hallermann, S., Pawlu, C., Jonas, P., and Heckmann, M. (2003). A large pool of releasable vesicles in a cortical glutamatergic synapse. Proc Natl Acad Sci U S A 100, 8975-8980.

Harrison, J., and Jahr, C.E. (2003). Receptor occupancy limits synaptic depression at climbing fiber synapses. J Neurosci 23, 377-383.

Hosoi, N., Sakaba, T., and Neher, E. (2007). Quantitative analysis of calcium-dependent vesicle recruitment and its functional role at the calyx of Held synapse. J Neurosci 27, 14286-14298.

Hubel, D.H., and Wiesel, T.N. (1962). Receptive fields, binocular interaction and functional architecture in the cat's visual cortex. J Physiol 160, 106-154.

Hubel, D.H., and Wiesel, T.N. (1965). Receptive Fields and Functional Architecture in Two Nonstriate Visual Areas (18 and 19) of the Cat. J Neurophysiol 28, 229-289. 
Isaacson, J.S., and Walmsley, B. (1995). Counting quanta: direct measurements of transmitter release at a central synapse. Neuron 15, 875-884.

Katz, B., and Miledi, R. (1965). The Measurement of Synaptic Delay, and the Time Course of Acetylcholine Release at the Neuromuscular Junction. Proc R Soc Lond B Biol Sci 161, 483-495.

Katz, B., and Miledi, R. (1967). The release of acetylcholine from nerve endings by graded electric pulses. Proc R Soc Lond B Biol Sci 167, 23-38.

Katz, B., and Miledi, R. (1972). The statistical nature of the acetycholine potential and its molecular components. J Physiol 224, 665-699.

Koester, H.J., and Johnston, D. (2005). Target cell-dependent normalization of transmitter release at neocortical synapses. Science 308, 863-866.

Korn, H., Triller, A., Mallet, A., and Faber, D.S. (1981). Fluctuating responses at a central synapse: $\mathrm{n}$ of binomial fit predicts number of stained presynaptic boutons. Science 213, 898-901.

Lefort, S., Tomm, C., Floyd Sarria, J.C., and Petersen, C.C. (2009). The excitatory neuronal network of the $\mathrm{C} 2$ barrel column in mouse primary somatosensory cortex. Neuron 61, 301-316.

Li, G.L., Keen, E., Andor-Ardo, D., Hudspeth, A.J., and von Gersdorff, H. (2009). The unitary event underlying multiquantal EPSCs at a hair cell's ribbon synapse. J Neurosci 29, 7558-7568.

Loebel, A., Silberberg, G., Helbig, D., Markram, H., Tsodyks, M., and Richardson, M.J. (2009). Multiquantal release underlies the distribution of synaptic efficacies in the neocortex. Front Comput Neurosci 3, 27. 
Lubke, J., Egger, V., Sakmann, B., and Feldmeyer, D. (2000). Columnar organization of dendrites and axons of single and synaptically coupled excitatory spiny neurons in layer 4 of the rat barrel cortex. J Neurosci 20, 5300-5311.

Mennerick, S., and Matthews, G. (1996). Ultrafast exocytosis elicited by calcium current in synaptic terminals of retinal bipolar neurons. Neuron 17, 1241-1249.

Meyer, A.C., Neher, E., and Schneggenburger, R. (2001). Estimation of quantal size and number of functional active zones at the calyx of held synapse by nonstationary EPSC variance analysis. J Neurosci 21, 7889-7900.

Moser, T., and Beutner, D. (2000). Kinetics of exocytosis and endocytosis at the cochlear inner hair cell afferent synapse of the mouse. Proc Natl Acad Sci U S A 97, 883-888.

Murphy, G.J., Glickfeld, L.L., Balsen, Z., and Isaacson, J.S. (2004). Sensory neuron signaling to the brain: properties of transmitter release from olfactory nerve terminals. J Neurosci 24, 3023-3030.

Murthy, V.N., Sejnowski, T.J., and Stevens, C.F. (1997). Heterogeneous release properties of visualized individual hippocampal synapses. Neuron 18, 599-612.

Neher, E., and Sakaba, T. (2001). Combining deconvolution and noise analysis for the estimation of transmitter release rates at the calyx of held. J Neurosci 21, 444-461.

Oertner, T.G., Sabatini, B.L., Nimchinsky, E.A., and Svoboda, K. (2002). Facilitation at single synapses probed with optical quantal analysis. Nat Neurosci 5, 657-664.

Pei, X., Vidyasagar, T.R., Volgushev, M., and Creutzfeldt, O.D. (1994). Receptive field analysis and orientation selectivity of postsynaptic potentials of simple cells in cat visual cortex. J Neurosci 14, 7130-7140. 
Petersen, C.C. (2002). Short-term dynamics of synaptic transmission within the excitatory neuronal network of rat layer 4 barrel cortex. J Neurophysiol 87, 2904-2914.

Petersen, C.C., and Sakmann, B. (2000). The excitatory neuronal network of rat layer 4 barrel cortex. J Neurosci 20, 7579-7586.

Petersen, C.C., and Sakmann, B. (2001). Functionally independent columns of rat somatosensory barrel cortex revealed with voltage-sensitive dye imaging. J Neurosci $21,8435-8446$.

Prinz, A.A., Abbott, L.F., and Marder, E. (2004). The dynamic clamp comes of age. Trends Neurosci 27, 218-224.

Rochefort, N.L., Garaschuk, O., Milos, R.I., Narushima, M., Marandi, N., Pichler, B., Kovalchuk, Y., and Konnerth, A. (2009). Sparsification of neuronal activity in the visual cortex at eye-opening. Proc Natl Acad Sci U S A 106, 15049-15054.

Rosenmund, C., Clements, J.D., and Westbrook, G.L. (1993). Nonuniform probability of glutamate release at a hippocampal synapse. Science 262, 754-757.

Rozov, A., Burnashev, N., Sakmann, B., and Neher, E. (2001). Transmitter release modulation by intracellular $\mathrm{Ca} 2+$ buffers in facilitating and depressing nerve terminals of pyramidal cells in layer $2 / 3$ of the rat neocortex indicates a target cell-specific difference in presynaptic calcium dynamics. J Physiol 531, 807-826.

Saviane, C., and Silver, R.A. (2006). Errors in the estimation of the variance: implications for multiple-probability fluctuation analysis. J Neurosci Methods 153, 250-260. 
Scheuss, V., Schneggenburger, R., and Neher, E. (2002). Separation of presynaptic and postsynaptic contributions to depression by covariance analysis of successive EPSCs at the calyx of held synapse. J Neurosci 22, 728-739.

Schikorski, T., and Stevens, C.F. (1997). Quantitative ultrastructural analysis of hippocampal excitatory synapses. J Neurosci 17, 5858-5867.

Silver, R.A., Lubke, J., Sakmann, B., and Feldmeyer, D. (2003). High-probability uniquantal transmission at excitatory synapses in barrel cortex. Science 302, 19811984.

Simons, D.J. (1978). Response properties of vibrissa units in rat SI somatosensory neocortex. J Neurophysiol 41, 798-820.

Singer, J.H., Lassova, L., Vardi, N., and Diamond, J.S. (2004). Coordinated multivesicular release at a mammalian ribbon synapse. Nat Neurosci 7, 826-833.

Stern, P., Edwards, F.A., and Sakmann, B. (1992). Fast and slow components of unitary EPSCs on stellate cells elicited by focal stimulation in slices of rat visual cortex. J Physiol 449, 247-278.

Stevens, C.F., and Wang, Y. (1995). Facilitation and depression at single central synapses. Neuron 14, 795-802.

Taschenberger, H., Leao, R.M., Rowland, K.C., Spirou, G.A., and von Gersdorff, H. (2002). Optimizing synaptic architecture and efficiency for high-frequency transmission. Neuron 36, 1127-1143.

Taschenberger, H., Scheuss, V., and Neher, E. (2005). Release kinetics, quantal parameters and their modulation during short-term depression at a developing synapse in the rat CNS. J Physiol 568, 513-537. 
Tong, G., and Jahr, C.E. (1994). Multivesicular release from excitatory synapses of cultured hippocampal neurons. Neuron 12, 51-59.

Trussell, L.O., Zhang, S., and Raman, I.M. (1993). Desensitization of AMPA receptors upon multiquantal neurotransmitter release. Neuron 10, 1185-1196.

Van der Kloot, W. (1988). The kinetics of quantal releases during end-plate currents at the frog neuromuscular junction. J Physiol 402, 605-626.

Verejone.D (1966). Simple Stochastic Models for Release of Quanta of Transmitter from a Nerve Terminal. Aust J Stat 8, 53-\&.

Wadiche, J.I., and Jahr, C.E. (2001). Multivesicular release at climbing fiber-Purkinje cell synapses. Neuron 32, 301-313.

Wang, L.Y., and Kaczmarek, L.K. (1998). High-frequency firing helps replenish the readily releasable pool of synaptic vesicles. Nature $394,384-388$.

Watanabe, J., Rozov, A., and Wollmuth, L.P. (2005). Target-specific regulation of synaptic amplitudes in the neocortex. J Neurosci 25, 1024-1033.

Welker, C. (1976). Receptive fields of barrels in the somatosensory neocortex of the rat. J Comp Neurol 166, 173-189.

Woolsey, T.A., and Van der Loos, H. (1970). The structural organization of layer IV in the somatosensory region (SI) of mouse cerebral cortex. The description of a cortical field composed of discrete cytoarchitectonic units. Brain Res 17, 205-242.

Wu, L.G., and Borst, J.G. (1999). The reduced release probability of releasable vesicles during recovery from short-term synaptic depression. Neuron 23, 821-832. 
Xu-Friedman, M.A., Harris, K.M., and Regehr, W.G. (2001). Three-dimensional comparison of ultrastructural characteristics at depressing and facilitating synapses onto cerebellar Purkinje cells. J Neurosci 21, 6666-6672.

Zucker, R.S. (1973). Changes in the statistics of transmitter release during facilitation. J Physiol 229, 787-810.

Zucker, R.S., and Regehr, W.G. (2002). Short-term synaptic plasticity. Annu Rev Physiol $64,355-405$. 


\section{Appendix}

\section{A.1 Abbreviations}

\begin{tabular}{ll} 
AMPAR & a-Amino-3-hydroxy-5-methyl-4-isoxazolepropionic acid receptor \\
AP & action potential \\
c.v. & coefficient of variance \\
D-AP5 & D(-)-2-amino-5-phosphonopentanoic acid \\
EPSC & excitatory post-synaptic current \\
FS & fast spiking neurons \\
GS & the conductance of S1 \\
GV & the conductance of V1 \\
Kyn & kynurenic acid \\
L2/3 & layer 2/3 \\
L4 & layer 4 \\
L5 & layer 5 \\
LTS & low-threshold spiking neurons \\
mEPSC & miniature excitatory post-synaptic current \\
ms & millisecond \\
MVR & multivesicular release \\
N & number of releasable units/ number of functional release sites \\
NMDAR & N-methyl-D-aspartic acid receptor \\
PPR & paired pulse ratio \\
Pr & release probability \\
q & the quantal size \\
RRP & readily releasable pool \\
RS & regular spiking neurons \\
S & second \\
S1 & somatosensory cortex \\
S.D. & standard deviation \\
TEA & tetraethylammonium \\
V1 & the primary visual cortex \\
V-M & variance-mean \\
& \\
\hline &
\end{tabular}




\section{Acknowledgement}

I would like to firstly thank my supervisor, Dr. Takeshi Sakaba, for his patient instruction which allowed me to have intensive and detailed discussion about the project and my future career. Furthermore, he always encourages me to express my own idea and opinion on my research, and gives me all his support for realizing the ideas. I am especially grateful for having such precious opportunity to build an independent attitude during my $\mathrm{PhD}$ period.

I am thankful to my committee members, Prof. Erwin Neher, Prof. Tobias Moser, and Dr. Oliver Schlueter for their continuous support and all the suggestions on my project during my $\mathrm{PhD}$ years.

I would like to show my gratitude to all members in the department of membrane biophysics and all members in the independent research group of synaptic transmission. Without their assistance I would not have finished my $\mathrm{PhD}$ so early and would not have such great time working in the department.

Special thanks are given to Dr. Kristian Wadel who trained me strictly during my initial phase working in this lab, and pushed me to develop a neat and precise habit of doing science. I also thank Dr. Jin Bao who was considerate enough to share a setup with me. She was very patient teaching me basic knowledge of programming and modeling, and conducted great discussion over our projects. Moreover, she developed dynamic clamp technique which is essential for my project and definitely pushed our publication to get through the review of the journal. It was a great time cooperating with her. I am very 
grateful to Lijun Yao, Raunak Sinha, and Kun-Han Lin. They supported me throughout these three years both scientifically and emotionally. With their company, working in the department became very cheery.

I would like to thank our department secretary, Irmgard Barteczko, for her great assistance on all kinds of administrative jobs. Also thanks to Sigrid Smidt, Frank Koehne, and Frank Wierriehausen for their excellent technical support.

I also would like to show my appreciation to the $\mathrm{MSc} / \mathrm{PhD}$ program of Neuroscience for offering me this invaluable chance to study in Germany, providing an extraordinary environment to expose myself to broad knowledge of neuroscience, and educating me to obtain a good appetite to do further research in this field. I thank Prof. Micheal Hoerner and Sandra Drube for taking really good care of students in the program, and helping me to solve problems in study and also in life. I would like to express my gratitude to Boehringer Ingelheim Fond for the generous financial support and offering a great community of outstanding scientists.

Lots of thanks to my most lovely classmates: Ramya Nair, Esra Fatma Demircioglu, Nikhil Sasidharan, Dario Arcos-Diaz, Shahaf Peleg, and all the others. They were with me throughout all the happiness, sadness, and difficulties within these four years of being in Goettingen. Without them, I would not have enjoyed my staying in Goettingen so much. I appreciate the Taiwanese community in Goettingen as well for the hearty help and the warm company.

In the end, I would like to thank my family and friends for their endless love and support which assured me that there is always a place for me so that I have the courage to accept all the challenges. 


\section{Curriculum Vitae}

\section{Personal Data}

Name: Chao-Hua Huang

Gender: Female

Date of birth: 02.10 .1983

Place of birth: Kaohsiung, Taiwan

Present Citizenship: Taiwan

\section{Education}

2001-2005: B.Sc. in Life Science (former Zoology department, zoology division) National Taiwan University, Taipei, Taiwan

2006-2007: International Max Planck Research School for Neurosciences (first segment) Georg August-University Göttingen, Germany

2007-2010: $\mathrm{PhD}$ thesis in the research group for biophysics of synaptic transmission at the Max Planck Institute for Biophysical Chemistry, Göttingen, Germany in the framework of the International Max Planck Research School for Neurosciences (second segment, $\mathrm{PhD}$ program) Georg August-University Göttingen, Germany 


\section{Publication list}

Chao-Hua Huang, Jin Bao, Takeshi Sakaba (2010). Multivesicular release differentiates the reliability of synaptic transmission between the visual cortex and the somatosensory cortex. J. Neuroscience, 30(36):11994-2004.

Chien-Yuan Pan, Chau-Hua Huang, Chia-Hsueh Lee (2006). Calcium elevation elicited by reverse mode $\mathrm{Na}^{+} / \mathrm{Ca}^{2+}$ exchange activity is facilitated by intracellular calcium stores in bovine chromaffin cells. Biochem Biophys Res Commun. 342(2):589-95. 